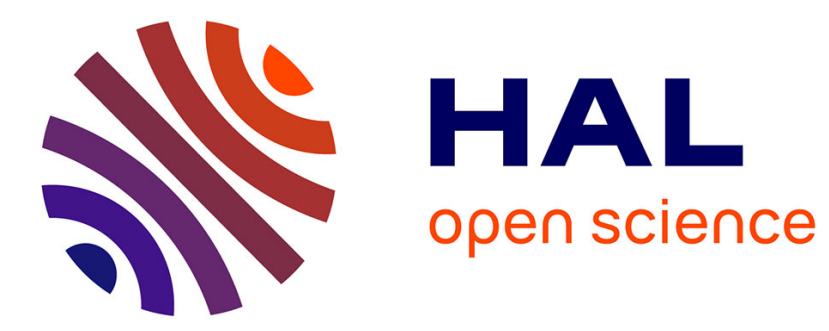

\title{
On a new formula relating localisation operators to time operators
}

Serge Richard, Rafael Tiedra de Aldecoa

\section{To cite this version:}

Serge Richard, Rafael Tiedra de Aldecoa. On a new formula relating localisation operators to time operators. Spectral Days 2010, 2010, Chile. pp.301 - 338. hal-00411376

\section{HAL Id: hal-00411376 \\ https://hal.science/hal-00411376}

Submitted on 27 Aug 2009

HAL is a multi-disciplinary open access archive for the deposit and dissemination of scientific research documents, whether they are published or not. The documents may come from teaching and research institutions in France or abroad, or from public or private research centers.
L'archive ouverte pluridisciplinaire HAL, est destinée au dépôt et à la diffusion de documents scientifiques de niveau recherche, publiés ou non, émanant des établissements d'enseignement et de recherche français ou étrangers, des laboratoires publics ou privés. 


\title{
On a new formula relating localisation operators to time operators
}

\author{
S. Richard ${ }^{1 *}$ and R. Tiedra de Aldecoa ${ }^{2}$ \\ ${ }^{1}$ Department of Pure Mathematics and Mathematical Statistics, Centre for Mathematical Sci- \\ ences, University of Cambridge, Cambridge, CB3 OWB, United Kingdom \\ ${ }^{2}$ Facultad de Matemáticas, Pontificia Universidad Católica de Chile, \\ Av. Vicuña Mackenna 4860, Santiago, Chile \\ E-mails:sr510@cam.ac.uk,rtiedra@mat.puc.cl
}

\begin{abstract}
We consider in a Hilbert space a self-adjoint operator $H$ and a family $\Phi \equiv\left(\Phi_{1}, \ldots, \Phi_{d}\right)$ of mutually commuting self-adjoint operators. Under some regularity properties of $H$ with respect to $\Phi$, we propose two new formulae for a time operator for $H$ and prove their equality. One of the expressions is based on the time evolution of an abstract localisation operator defined in terms of $\Phi$ while the other one corresponds to a stationary formula. Under the same assumptions, we also conduct the spectral analysis of $H$ by using the method of the conjugate operator.

Among other examples, our theory applies to Friedrichs Hamiltonians, Stark Hamiltonians, some Jacobi operators, the Dirac operator, convolution operators on locally compact groups, pseudodifferential operators, adjacency operators on graphs and direct integral operators.
\end{abstract}

2000 Mathematics Subject Classification: 46N50, 81Q10, 47A40.

\section{Introduction and main results}

Let $H$ be a self-adjoint operator in a Hilbert space $\mathcal{H}$ and let $T$ be a linear operator in $\mathcal{H}$. Generally speaking, the operator $T$ is called a time operator for $H$ if it satisfies the canonical commutation relation

$$
[T, H]=i,
$$

or, alternatively, the relation

$$
T \mathrm{e}^{-i t H}=\mathrm{e}^{-i t H}(T+t) .
$$

Obviously, these two equations are very formal and not equivalent. So many authors have proposed various sets of conditions in order to give a precise meaning to them. For instance, one has introduced the concept of infinitesimal Weyl relation in the weak or in the strong sense [18], the $T$-weak Weyl relation [19] or various generalised versions of the Weyl relation (see e.g. $[6,17])$. However, in most of these publications the pair $\{H, T\}$ is a priori given and the authors are mainly interested in the properties of $H$ and $T$ that can be deduced from a relation like (1.2). In particular, the self-adjointness of $T$, the spectral nature of $H$ and $T$, the connection with the survival probability, the form of $T$ in the spectral representation of $H$, the relation with the theory of irreversibility and many other properties have been extensively discussed in the literature (see [23, Sec. 8], [24, Sec. 3], [5, 12, 14, 16, 39] and references therein).

\footnotetext{
*On leave from Université de Lyon; Université Lyon 1; CNRS, UMR5208, Institut Camille Jordan, 43 blvd du 11 novembre 1918, F-69622 Villeurbanne-Cedex, France.
} 
Our approach is radically different. Starting from a self-adjoint operator $H$, one wonders if there exists a linear operator $T$ such that (1.1) holds in a suitable sense. And can we find a universal procedure to construct such an operator? This paper is a first attempt to answer these questions.

Our interest in these questions has been recently aroused by a formula put into evidence in [37]. Along the proof of the existence of time delay for hypoelliptic pseudodifferential operators $H:=h(P)$ in $\mathrm{L}^{2}\left(\mathbb{R}^{d}\right)$, the author derives an integral formula linking the time evolution of localisation operators to the derivative with respect to the spectral parameter of $H$. The formula reads as follows: if $Q$ stands for the family of position operators in $\mathrm{L}^{2}\left(\mathbb{R}^{d}\right)$ and $f: \mathbb{R}^{d} \rightarrow \mathbb{C}$ is some appropriate function with $f=1$ in a neighbourhood of 0 , then one has on suitable elements $\varphi \in \mathrm{L}^{2}\left(\mathbb{R}^{d}\right)$

$$
\lim _{r \rightarrow \infty} \frac{1}{2} \int_{0}^{\infty} \mathrm{d} t\left\langle\varphi,\left[\mathrm{e}^{-i t H} f(Q / r) \mathrm{e}^{i t H}-\mathrm{e}^{i t H} f(Q / r) \mathrm{e}^{-i t H}\right] \varphi\right\rangle=\left\langle\varphi, i \frac{\mathrm{d}}{\mathrm{d} H} \varphi\right\rangle
$$

where $\frac{\mathrm{d}}{\mathrm{d} H}$ stands for the operator acting as $\frac{\mathrm{d}}{\mathrm{d} \lambda}$ in the spectral representation of $H$. So, this formula furnishes a standardized procedure to obtain a time operator $T$ only constructed in terms of $H$, the position operators $Q$ and the function $f$.

A review of the methods used in [37] suggested to us that Equation (1.3) could be extended to the case of an abstract pair of operator $H$ and position operators $\Phi$ acting in a Hilbert space $\mathcal{H}$, as soon as $H$ and $\Phi$ satisfy two appropriate commutation relations. Namely, suppose that you are given a self-adjoint operator $H$ and a family $\Phi \equiv\left(\Phi_{1}, \ldots, \Phi_{d}\right)$ of mutually commuting self-adjoint operators in $\mathcal{H}$. Then, roughly speaking, the first condition requires that for some $\omega \in \mathbb{C} \backslash \mathbb{R}$ the map

$$
\mathbb{R}^{d} \ni x \mapsto \mathrm{e}^{-i x \cdot \Phi}(H-\omega)^{-1} \mathrm{e}^{i x \cdot \Phi} \in \mathscr{B}(\mathcal{H})
$$

is 3-times strongly differentiable (see Assumption 2.2 for a precise statement). The second condition, Assumption 2.3 , requires that for each $x \in \mathbb{R}^{d}$, the operators $\mathrm{e}^{-i x \cdot \Phi} H \mathrm{e}^{i x \cdot \Phi}$ mutually commute. Given this, our main result reads as follows (see Theorem 5.5 for a precise statement):

Theorem 1.1. Let $H$ and $\Phi$ be as above. Let $f$ be a Schwartz function on $\mathbb{R}^{d}$ such that $f=1$ on a neighbourhood of 0 and $f(x)=f(-x)$ for each $x \in \mathbb{R}^{d}$. Then, for each $\varphi$ in some suitable subset of $\mathcal{H}$ one has

$$
\lim _{r \rightarrow \infty} \frac{1}{2} \int_{0}^{\infty} \mathrm{d} t\left\langle\varphi,\left[\mathrm{e}^{-i t H} f(\Phi / r) \mathrm{e}^{i t H}-\mathrm{e}^{i t H} f(\Phi / r) \mathrm{e}^{-i t H}\right] \varphi\right\rangle=\left\langle\varphi, T_{f} \varphi\right\rangle,
$$

where the operator $T_{f}$ acts, in an appropriate sense, as $i \frac{\mathrm{d}}{\mathrm{d} \lambda}$ in the spectral representation of $H$.

One infers from this result that the operator $T_{f}$ is a time operator. Furthermore, an explicit description of $T_{f}$ is also available: if $H_{j}^{\prime}$ denotes the self-adjoint operator associated with the commutator $i\left[H, \Phi_{j}\right]$ and $H^{\prime}:=\left(H_{1}^{\prime}, \ldots, H_{d}^{\prime}\right)$, then $T_{f}$ is formally given by

$$
T_{f}=-\frac{1}{2}\left(\Phi \cdot R_{f}^{\prime}\left(H^{\prime}\right)+R_{f}^{\prime}\left(H^{\prime}\right) \cdot \Phi\right),
$$

where $R_{f}^{\prime}: \mathbb{R}^{d} \rightarrow \mathbb{C}^{d}$ is some explicit function (see Section 4 and Proposition 5.2).

In summary, once a family of mutually commuting self-adjoint operators $\left(\Phi_{1}, \ldots, \Phi_{d}\right)$ satisfying Assumptions 2.2 and 2.3 has been given, then a time operator can be defined either in terms of the 1.h.s. of (1.4) or in terms of (1.5). When suitably defined, both expressions lead to the same operator. We also mention that the equality (1.4), with r.h.s. defined by (1.5), provides a crucial preliminary step for the proof of the existence of quantum time delay and Eisenbud-Wigner Formula for abstract scattering pairs $\{H, H+V\}$. In addition, Theorem 1.1 establishes a new relation between time dependent scattering theory (1.h.s.) and stationary scattering theory (r.h.s.) for a general class of operators. We refer to the discussion in Section 6 for more information on these issues.

Let us now describe more precisely the content of this paper. In Section 2 we recall the necessary definitions from the theory of the conjugate operator and define a critical set $\kappa(H)$ for the operator $H$. In the more usual 
setup where $H=h(P)$ is a function of the momentum vector operator $P$ and $\Phi$ is the position vector operator $Q$ in $\mathrm{L}^{2}\left(\mathbb{R}^{d}\right)$, it is known that the critical values of $h$

$$
\kappa_{h}:=\left\{\lambda \in \mathbb{R} \mid \exists x \in \mathbb{R}^{d} \text { such that } h(x)=\lambda \text { and } h^{\prime}(x)=0\right\}
$$

plays an important role (see e.g. [1, Sec. 7]). Typically, the operator $h(P)$ has bad spectral properties and bad propagation properties on $\kappa_{h}$. For instance, one cannot obtain a simple Mourre estimate at these values. Such phenomena also occur in the abstract setup. Since the operator $H$ is a priori not a function of an auxiliary operator as $h(P)$, the derivative appearing in the definition of $\kappa_{h}$ does not have a direct counterpart. However, the identities $\left(\partial_{j} h\right)(P)=i\left[h(P), Q_{j}\right]$ suggest to define the set of critical values $\kappa(H)$ in terms of the vector operator $H^{\prime}:=\left(i\left[H, \Phi_{1}\right], \ldots, i\left[H, \Phi_{d}\right]\right)$. This is the content of Definition 2.5. In Lemma 2.6 and Theorem 3.6, we show that $\kappa(H)$ is closed, contains the set of eigenvalues of $H$, and that the spectrum of $H$ in $\sigma(H) \backslash \kappa(H)$ is purely absolutely continuous. The proof of the latter result relies on the construction, described in Section 3 , of an appropriate conjugate operator for $H$.

In Section 4, we recall some definitions in relation with the function $f$ that appear in Theorem 1.1. The function $R_{f}$ is introduced and some of its properties are presented. Section 5 is the core of the paper and its most technical part. It contains the definition of $T_{f}$ and the proof of the precise version of Theorem 1.1. Suitable subspaces of $\mathcal{H}$ on which the operators are well-defined and on which the equalities hold are introduced.

An interpretation of our results is proposed in Section 6. The relation with the theory of time operators is explained, and various cases are presented. The importance of Theorem 5.5 for the proof of the existence of the quantum time delay and Eisenbud-Wigner Formula is also sketched.

In Section 7, we show that our results apply to many operators $H$ appearing in physics and mathematics literature. Among other examples, we treat Friedrichs Hamiltonians, Stark Hamiltonians, some Jacobi operators, the Dirac operator, convolution operators on locally compact groups, pseudodifferential operators, adjacency operators on graphs and direct integral operators. In each case, we are able to exhibit a natural family of position operators $\Phi$ satisfying our assumptions. The diversity of the examples covered by our theory make us strongly believe that Formula (1.4) is of natural character. Moreover it also suggests that the existence of time delay is a very common feature of quantum scattering theory. We also point out that one by-product of our study is an efficient algorithm for the choice of a conjugate operator for a given self-adjoint operator $H$ (see Section 3). This allows us to obtain (or reobtain) non trivial spectral results for various important classes of self-adjoint operators $H$.

As a final comment, we would like to emphasize that one of the main interest of our study comes from the fact that we do not restrict ourselves to the standard position operators $Q$ and to operators $H$ which are functions of $P$. Due to this generality, we cannot rely on the usual canonical commutation relation of $Q$ and $P$ and on the subjacent Fourier analysis. This explains the constant use of abstract commutators methods throughout the paper.

\section{Critical values}

In this section, we recall some standard notions on the conjugate operator theory and introduce our general framework. The set of critical values is defined and some of its properties are outlined. This subset of the spectrum of the operator under investigation plays an essential role in the sequel.

We first recall some facts principally borrowed from [1]. Let $H$ and $A$ be two self-adjoint operators in a Hilbert space $\mathcal{H}$. Their respective domain are denoted by $\mathcal{D}(H)$ and $\mathcal{D}(A)$, and for suitable $\omega \in \mathbb{C}$ we write $R_{\omega}$ for $(H-\omega)^{-1}$. The operator $H$ is of class $C^{1}(A)$ if there exists $\omega \in \mathbb{C} \backslash \sigma(H)$ such that the map

$$
\mathbb{R} \ni t \mapsto \mathrm{e}^{-i t A} R_{\omega} \mathrm{e}^{i t A} \in \mathscr{B}(\mathcal{H})
$$

is strongly differentiable. In that case, the quadratic form

$$
\mathcal{D}(A) \ni \varphi \mapsto\left\langle A \varphi, R_{\omega} \varphi\right\rangle-\left\langle R_{\omega}^{*} \varphi, A \varphi\right\rangle \in \mathbb{C}
$$

extends continuously to a bounded operator denoted by $\left[A, R_{\omega}\right] \in \mathscr{B}(\mathcal{H})$. It also follows from the $C^{1}(A)$ condition that $\mathcal{D}(H) \cap \mathcal{D}(A)$ is a core for $H$ and that the quadratic form $\mathcal{D}(H) \cap \mathcal{D}(A) \ni \varphi \mapsto\langle H \varphi, A \varphi\rangle-$ 
$\langle A \varphi, H \varphi\rangle$ is continuous in the topology of $\mathcal{D}(H)$. This form extends then uniquely to a continuous quadratic form $[H, A]$ on $\mathcal{D}(H)$, which can be identified with a continuous operator from $\mathcal{D}(H)$ to $\mathcal{D}(H)^{*}$. Finally, the following equality holds:

$$
\left[A, R_{\omega}\right]=R_{\omega}[H, A] R_{\omega} .
$$

It is also proved in [13, Lemma 2] that if $[H, A] \mathcal{D}(H) \subset \mathcal{H}$, then the unitary group $\left\{\mathrm{e}^{i t A}\right\}_{t \in \mathbb{R}}$ preserves the domain of $H$, i.e. $\mathrm{e}^{i t A} \mathcal{D}(H) \subset \mathcal{D}(H)$ for all $t \in \mathbb{R}$.

We now extend this framework in two directions: in the number of conjugate operators and in the degree of regularity with respect to these operators. So, let us consider a family $\Phi \equiv\left(\Phi_{1}, \ldots, \Phi_{d}\right)$ of mutually commuting self-adjoint operators in $\mathcal{H}$ (throughout the paper, we use the term "commute" for operators commuting in the sense of [26, Sec. VIII.5]). Then we know from [7, Sec. 6.5] that any measurable function $f \in \mathrm{L}^{\infty}\left(\mathbb{R}^{d}\right)$ defines a bounded operator $f(\Phi)$ in $\mathcal{H}$. In particular, the operator $\mathrm{e}^{i x \cdot \Phi}$, with $x \cdot \Phi \equiv \sum_{j=1}^{d} x_{j} \Phi_{j}$, is unitary for each $x \in \mathbb{R}^{d}$. Note also that the conjugation

$$
C_{x}: \mathscr{B}(\mathcal{H}) \rightarrow \mathscr{B}(\mathcal{H}), \quad B \mapsto \mathrm{e}^{-i x \cdot \Phi} B \mathrm{e}^{i x \cdot \Phi}
$$

defines an automorphism of $\mathscr{B}(\mathcal{H})$.

Within this framework, the operator $H$ is said to be of class $C^{m}(\Phi)$ for $m=1,2, \ldots$ if there exists $\omega \in \mathbb{C} \backslash \sigma(H)$ such that the map

$$
\mathbb{R}^{d} \ni x \mapsto \mathrm{e}^{-i x \cdot \Phi} R_{\omega} \mathrm{e}^{i x \cdot \Phi} \in \mathscr{B}(\mathcal{H})
$$

is strongly of class $C^{m}$ in $\mathcal{H}$. One easily observes that if $H$ is of class $C^{m}(\Phi)$, then the operator $H$ is of class $C^{m}\left(\Phi_{j}\right)$ for each $j$ (the class $C^{m}\left(\Phi_{j}\right)$ being defined similarly).

Remark 2.1. A bounded operator $S \in \mathscr{B}(\mathcal{H})$ belongs to $C^{1}(A)$ if the map (2.1), with $R_{\omega}$ replaced by $S$, is strongly differentiable. Similarly, $S \in \mathscr{B}(\mathcal{H})$ belongs to $C^{m}(\Phi)$ if the map (2.3), with $R_{\omega}$ replaced by $S$, is strongly $C^{m}$.

In the sequel, we assume that $H$ is regular with respect to unitary group $\left\{\mathrm{e}^{i x \cdot \Phi}\right\}_{x \in \mathbb{R}^{d}}$ in the following sense.

Assumption 2.2. The operator $H$ is of class $C^{3}(\Phi)$. Furthermore, for each $j \in\{1, \ldots, d\}$, the quadratic form $i\left[H, \Phi_{j}\right]$ on $\mathcal{D}(H)$ defines an essentially self-adjoint operator whose self-adjoint extension is denoted by $H_{j}^{\prime}$. Similarly, for each $k, \ell \in\{1, \ldots, d\}$, the quadratic form $i\left[H_{j}^{\prime}, \Phi_{k}\right]$ on $\mathcal{D}\left(H_{j}^{\prime}\right)$ defines an essentially self-adjoint operator whose self-adjoint extension is denoted by $H_{j k}^{\prime \prime}$, and the quadratic form $i\left[H_{j k}^{\prime \prime}, \Phi_{\ell}\right]$ on $\mathcal{D}\left(H_{j k}^{\prime \prime}\right)$ defines an essentially self-adjoint operator whose self-adjoint extension is denoted by $H_{j k \ell}^{\prime \prime \prime}$.

This assumption implies the invariance of $\mathcal{D}(H)$ under the action of the unitary group $\left\{\mathrm{e}^{i x \cdot \Phi}\right\}_{x \in \mathbb{R}^{d}}$. Indeed, if the quadratic form $i\left[H, \Phi_{j}\right]$ on $\mathcal{D}(H)$ defines an essentially self-adjoint operator in $\mathcal{H}$, it follows in particular that $\mathcal{D}(H) \subset \mathcal{D}\left(H_{j}^{\prime}\right)$ and thus $i\left[H, \Phi_{j}\right] \mathcal{D}(H) \equiv H_{j}^{\prime} \mathcal{D}(H) \subset \mathcal{H}$. It follows then from [13, Lemma 2] that $\mathrm{e}^{i t \Phi_{j}} \mathcal{D}(H) \subset \mathcal{D}(H)$ for all $t \in \mathbb{R}$. In fact, one easily obtains that $\mathrm{e}^{i t \Phi_{j}} \mathcal{D}(H)=\mathcal{D}(H)$, and since this property holds for each $j$ one also has $\mathrm{e}^{i x \cdot \Phi} \mathcal{D}(H)=\mathcal{D}(H)$ for all $x \in \mathbb{R}^{d}$. As a consequence, we obtain in particular that each self-adjoint operator

$$
H(x):=\mathrm{e}^{-i x \cdot \Phi} H \mathrm{e}^{i x \cdot \Phi}
$$

(with $H(0)=H$ ) has domain $\mathcal{D}[H(x)]=\mathcal{D}(H)$.

Similarly, the domains $\mathcal{D}\left(H_{j}^{\prime}\right)$ and $\mathcal{D}\left(H_{j k}^{\prime \prime}\right)$ are left invariant by the action of the unitary group $\left\{\mathrm{e}^{i x \cdot \Phi}\right\}_{x \in \mathbb{R}^{d}}$, and the operators $H_{j}^{\prime}(x):=\mathrm{e}^{-i x \cdot \Phi} H_{j}^{\prime} \mathrm{e}^{i x \cdot \Phi}$ and $H_{j k}^{\prime \prime}(x):=\mathrm{e}^{-i x \cdot \Phi} H_{j k}^{\prime \prime} \mathrm{e}^{i x \cdot \Phi}$ are self-adjoint operators with domains $\mathcal{D}\left(H_{j}^{\prime}\right)$ and $\mathcal{D}\left(H_{j k}^{\prime \prime}\right)$ respectively.

Our second main assumption concerns the family of operators $H(x)$.

Assumption 2.3. The operators $\{H(x)\}_{x \in \mathbb{R}^{d}}$ mutually commute.

Using the fact that the map $\mathbb{R}^{d} \ni x \mapsto C_{x} \in \operatorname{Aut}[\mathscr{B}(\mathcal{H})]$ is a group morphism, one easily shows that Assumption 2.3 is equivalent the commutativity of each $H(x)$ with $H$. Furthermore, Assumptions 2.2 and 2.3 imply additional commutation relations: 
Lemma 2.4. The operators $H(x), H_{j}^{\prime}(y), H_{k \ell}^{\prime \prime}(z)$ mutually commute for each $j, k, \ell \in\{1, \ldots, d\}$ and each $x, y, z \in \mathbb{R}^{d}$.

Proof. Let $\omega \in \mathbb{C} \backslash \mathbb{R}, x, y, z \in \mathbb{R}^{d}, j, k, \ell, m \in\{1, \ldots, d\}$, and set $R(x):=[H(x)-\omega]^{-1}, R_{j}^{\prime}(x):=$ $\left[H_{j}^{\prime}(x)-\omega\right]^{-1}$ and $R_{j k}^{\prime \prime}(x):=\left[H_{j k}^{\prime \prime}(x)-\omega\right]^{-1}$. By assumption, one has the equality

$$
R(x) \frac{R\left(\varepsilon e_{j}\right)-R(0)}{\varepsilon}=\frac{R\left(\varepsilon e_{j}\right)-R(0)}{\varepsilon} R(x)
$$

for each $\varepsilon \in \mathbb{R} \backslash\{0\}$. Taking the strong limit as $\varepsilon \rightarrow 0$, and using (2.2) and Assumption 2.3, one obtains

$$
R(0)\left[R(x) H_{j}^{\prime}-H_{j}^{\prime} R(x)\right] R(0)=0 .
$$

Since the resolvent $R(0)$ on the left is injective, this implies that $R(x) H_{j}^{\prime}-H_{j}^{\prime} R(x)=0$ on $\mathcal{D}(H)$. Furthermore, since $\mathcal{D}(H)$ is a core for $H_{j}^{\prime}$ the last equality can be extended to $\mathcal{D}\left(H_{j}^{\prime}\right)$. Finally, by multiplying the equation

$$
R(x)=R(x)\left(H_{j}^{\prime}-\omega\right) R_{j}^{\prime}(0)=\left(H_{j}^{\prime}-\omega\right) R(x) R_{j}^{\prime}(0)
$$

on the left by $R_{j}^{\prime}(0)$, one gets $R_{j}^{\prime}(0) R(x)=R(x) R_{j}^{\prime}(0)$. Using the morphism property of the map $\mathbb{R}^{d} \ni x \mapsto$ $C_{x} \in \operatorname{Aut}[\mathscr{B}(\mathcal{H})]$, one infers from this that $H(x)$ and $H_{j}^{\prime}(y)$ commute.

A similar argument leads to the commutativity of the operators $H_{j}^{\prime}(x)$ and $H_{k}^{\prime}(y)$ by considering the operators $R_{j}^{\prime}(x) \frac{R\left(\varepsilon e_{k}\right)-R(0)}{\varepsilon}$ and $\frac{R\left(\varepsilon e_{k}\right)-R(0)}{\varepsilon} R_{j}^{\prime}(x)$. The commutativity of $H(x)$ and $H_{j k}^{\prime \prime}(z)$ is obtained by considering the operators $R(x) \frac{R_{j}^{\prime}\left(\varepsilon e_{k}\right)-R_{j}^{\prime}(0)}{\varepsilon}$ and $\frac{R_{j}^{\prime}\left(\varepsilon e_{k}\right)-R_{j}^{\prime}(0)}{\varepsilon} R(x)$, and the commutativity of $H_{j}^{\prime}(y)$ and $H_{k \ell}^{\prime \prime}(z)$ by considering the operators $R_{j}^{\prime}(y) \frac{R_{k}^{\prime}\left(\varepsilon e_{\ell}\right)-R_{k}^{\prime}(0)}{\varepsilon}$ and $\frac{R_{k}^{\prime}\left(\varepsilon e_{\ell}\right)-R_{k}^{\prime}(0)}{\varepsilon} R_{j}^{\prime}(y)$. Finally, the commutation between $H_{j k}^{\prime \prime}(x)$ and $H_{\ell m}^{\prime \prime}(y)$ is obtained by considering the operators $R_{j k}^{\prime \prime}(x) \frac{R_{\ell}^{\prime}\left(\varepsilon e_{m}\right)-R_{\ell}^{\prime}(0)}{\varepsilon}$ and $\frac{R_{\ell}^{\prime}\left(\varepsilon e_{m}\right)-R_{\ell}^{\prime}(0)}{\varepsilon} R_{j k}^{\prime \prime}(x)$. Details are left to the reader.

For simplicity, we write $H^{\prime}$ for the vector operator $\left(H_{1}^{\prime}, \ldots, H_{d}^{\prime}\right)$, and define for each measurable function $f: \mathbb{R}^{d} \rightarrow \mathbb{C}$ the operator $f\left(H^{\prime}\right)$ by using the $d$-variables functional calculus. The symbol $E^{H}(\cdot)$ denotes the spectral measure of $H$.

Definition 2.5. A number $\lambda \in \mathbb{R}$ is called a regular value of $H$ if there exists $\delta>0$ such that

$$
\lim _{\varepsilon \searrow 0}\left\|\left[\left(H^{\prime}\right)^{2}+\varepsilon\right]^{-1} E^{H}((\lambda-\delta, \lambda+\delta))\right\|<\infty .
$$

A number $\lambda \in \mathbb{R}$ that is not a regular value of $H$ is called a critical value of $H$. We denote by $\kappa(H)$ the set of critical values of $H$.

From now on, we shall use the shorter notation $E^{H}(\lambda ; \delta)$ for $E^{H}((\lambda-\delta, \lambda+\delta))$. In the next lemma we put into evidence some useful properties of the set $\kappa(H)$.

Lemma 2.6. Let Assumptions 2.2 and 2.3 be verified. Then the set $\kappa(H)$ possesses the following properties:

(a) $\kappa(H)$ is closed.

(b) $\kappa(H)$ contains the set of eigenvalues of $H$.

(c) The limit $\lim _{\varepsilon \backslash 0}\left\|\left[\left(H^{\prime}\right)^{2}+\varepsilon\right]^{-1} E^{H}(J)\right\|$ is finite for each compact set $J \subset \mathbb{R} \backslash \kappa(H)$.

(d) For each compact set $J \subset \mathbb{R} \backslash \kappa(H)$, there exists a compact set $U \subset(0, \infty)$ such that $E^{H}(J)=$ $E^{\left|H^{\prime}\right|}(U) E^{H}(J)$.

Proof. (a) Let $\lambda_{0}$ be a regular value for $H$, i.e. there exists $\delta_{0}>0$ such that (2.5) holds with $\delta$ replaced by $\delta_{0}$. Let $\lambda \in\left(\lambda_{0}-\delta_{0}, \lambda_{0}+\delta_{0}\right)$ and let $\delta>0$ such that

$$
(\lambda-\delta, \lambda+\delta) \subset\left(\lambda_{0}-\delta_{0}, \lambda_{0}+\delta_{0}\right)
$$


Then, since $E^{H}(\lambda ; \delta)=E^{H}\left(\lambda_{0} ; \delta_{0}\right) E^{H}(\lambda ; \delta)$, one has

$$
\lim _{\varepsilon \searrow 0}\left\|\left[\left(H^{\prime}\right)^{2}+\varepsilon\right]^{-1} E^{H}(\lambda ; \delta)\right\| \leq \lim _{\varepsilon \searrow 0}\left\|\left[\left(H^{\prime}\right)^{2}+\varepsilon\right]^{-1} E^{H}\left(\lambda_{0} ; \delta_{0}\right)\right\|<\infty .
$$

But this means exactly that $\lambda$ is a regular value for any $\lambda \in\left(\lambda_{0}-\delta_{0}, \lambda_{0}+\delta_{0}\right)$. So the set of regular values is open, and $\kappa(H)$ is closed.

(b) Let $\lambda \in \mathbb{R}$ be an eigenvalue of $H$, and let $\varphi_{\lambda}$ be an associated eigenvector with norm one. Since $H$ is of class $C^{1}\left(\Phi_{j}\right)$ for each $j$, we know from the Virial theorem [1, Prop. 7.2.10] that $E^{H}(\{\lambda\}) H_{j}^{\prime} E^{H}(\{\lambda\})=0$ for each $j$. This, together with Lemma 2.4, implies that

$$
E^{H}(\{\lambda\})\left[\left(H^{\prime}\right)^{2}+\varepsilon\right]^{-1} E^{H}(\{\lambda\})=\varepsilon^{-1} E^{H}(\{\lambda\})
$$

for each $\varepsilon>0$. In particular, we obtain for each $\delta>0$ the equalities

$$
\left[\left(H^{\prime}\right)^{2}+\varepsilon\right]^{-1} E^{H}(\lambda ; \delta) \varphi_{\lambda}=E^{H}(\{\lambda\})\left[\left(H^{\prime}\right)^{2}+\varepsilon\right]^{-1} E^{H}(\{\lambda\}) \varphi_{\lambda}=\varepsilon^{-1} \varphi_{\lambda},
$$

and

$$
\lim _{\varepsilon \searrow 0}\left\|\left[\left(H^{\prime}\right)^{2}+\varepsilon\right]^{-1} E^{H}(\lambda ; \delta)\right\| \geq \lim _{\varepsilon \searrow 0}\left\|\left[\left(H^{\prime}\right)^{2}+\varepsilon\right]^{-1} E^{H}(\lambda ; \delta) \varphi_{\lambda}\right\|=\lim _{\varepsilon \searrow 0} \varepsilon^{-1}\left\|\varphi_{\lambda}\right\|=\infty .
$$

Since $\delta$ has been chosen arbitrarily, this implies that $\lambda$ is not a regular value of $H$.

(c) This follows easily by using a compacity argument.

(d) Let us concentrate first on the lower bound of $U$. Clearly, if $\left|H^{\prime}\right|$ is strictly positive, then $U$ can be chosen in $(0, \infty)$ and thus is bounded from below by a strictly positive number. So assume now that $\left|H^{\prime}\right|$ is not strictly positive, that is $0 \in \sigma\left(\left|H^{\prime}\right|\right)$. By absurd, suppose that $U$ is not bounded from below by a strictly positive number, i.e. there does not exist $a>0$ such that $U \subset(a, \infty)$. Then for $n=1,2, \ldots$, there exists $\psi_{n} \in \mathcal{H}$ such that $E^{\left|H^{\prime}\right|}([0,1 / n)) E^{H}(J) \psi_{n} \neq 0$, and the vectors

$$
\varphi_{n}:=\frac{E^{\left|H^{\prime}\right|}([0,1 / n)) E^{H}(J) \psi_{n}}{\left\|E^{\left|H^{\prime}\right|}([0,1 / n)) E^{H}(J) \psi_{n}\right\|}
$$

satisfy $\left\|\varphi_{n}\right\|=1$, and $E^{H}(J) \varphi_{n}=E^{\left|H^{\prime}\right|}([0,1 / n)) \varphi_{n}=\varphi_{n}$. It follows by point (c) that

$$
\begin{aligned}
\text { Const. } \geq \lim _{\varepsilon \searrow 0}\left\|\left[\left(H^{\prime}\right)^{2}+\varepsilon\right]^{-1} E^{H}(J)\right\| & \geq \lim _{\varepsilon \searrow 0}\left\|\left[\left(H^{\prime}\right)^{2}+\varepsilon\right]^{-1} E^{H}(J) \varphi_{n}\right\| \\
& =\lim _{\varepsilon \searrow 0}\left\|\left[\left(H^{\prime}\right)^{2}+\varepsilon\right]^{-1} E^{\left|H^{\prime}\right|}([0,1 / n)) \varphi_{n}\right\| \\
& \geq \lim _{\varepsilon \searrow 0}\left(n^{-2}+\varepsilon\right)^{-1}\left\|\varphi_{n}\right\| \\
& =n^{2},
\end{aligned}
$$

which leads to a contradiction when $n \rightarrow \infty$.

Let us now concentrate on the upper bound of $U$. Clearly, if $\left|H^{\prime}\right|$ is a bounded operator, one can choose a bounded subset $U$ of $\mathbb{R}$ and thus $U$ is upper bounded. So assume now that $\left|H^{\prime}\right|$ is not a bounded operator. By absurd, suppose that $U$ is not bounded from above, i.e. there does not exist $b<\infty$ such that $U \subset(0, b)$. Then for $n=1,2, \ldots$, there exists $\psi_{n} \in \mathcal{H}$ such that $E^{\left|H^{\prime}\right|}([n, \infty)) E^{H}(J) \psi_{n} \neq 0$, and the vectors

$$
\varphi_{n}:=\frac{E^{\left|H^{\prime}\right|}([n, \infty)) E^{H}(J) \psi_{n}}{\left\|E^{\left|H^{\prime}\right|}([n, \infty)) E^{H}(J) \psi_{n}\right\|}
$$

satisfy $\left\|\varphi_{n}\right\|=1$, and $E^{H}(J) \varphi_{n}=E^{\left|H^{\prime}\right|}([n, \infty)) \varphi_{n}=\varphi_{n}$. It follows by Assumption 2.2 and Lemma 2.4 that $\left|H^{\prime}\right| E^{H}(J)$ is a bounded operator, and

$$
\text { Const. } \geq\left\|\left|H^{\prime}\right| E^{H}(J)\right\| \geq\left\|\left|H^{\prime}\right| E^{H}(J) \varphi_{n}\right\|=\left\|\left|H^{\prime}\right| E^{\left|H^{\prime}\right|}([n, \infty)) \varphi_{n}\right\| \geq n\left\|\varphi_{n}\right\|
$$

which leads to a contradiction when $n \rightarrow \infty$. 


\section{Locally smooth operators and absolute continuity}

In this section we exhibit a large class of locally $H$-smooth operators. We also show that the operator $H$ is purely absolutely continuous in $\sigma(H) \backslash \kappa(H)$. These results are obtained by using commutators methods as presented in [1].

In order to motivate our choice of conjugate operator for $H$, we present first a formal calculation. Let $A_{\eta}$ be given by

$$
A_{\eta}:=\frac{1}{2}\left\{\eta(H) H^{\prime} \cdot \Phi+\Phi \cdot H^{\prime} \eta(H)\right\},
$$

where $\eta$ is some real function with a sufficiently rapid decrease to 0 at infinity. Then $A_{\eta}$ satisfies with $H$ the commutation relation

$$
i\left[H, A_{\eta}\right]=\frac{i}{2} \sum_{j=1}^{d}\left\{\eta(H) H_{j}^{\prime}\left[H, \Phi_{j}\right]+\left[H, \Phi_{j}\right] H_{j}^{\prime} \eta(H)\right\}=\left(H^{\prime}\right)^{2} \eta(H)
$$

which provides (in a sense to be specified) a Mourre estimate. So, in the sequel, one only has to justify these formal manipulations and to determinate an appropriate function $\eta$.

First of all, one observes that for each $j \in\{1, \ldots, d\}$ and each $\omega \in \mathbb{C} \backslash \sigma(H)$ the operator $H_{j}^{\prime} R_{\omega} \equiv$ $H_{j}^{\prime}(H-\omega)^{-1}$ is a bounded operator. Indeed, one has $(H-\omega)^{-1} \mathcal{H}=\mathcal{D}(H) \subset \mathcal{D}\left(H_{j}^{\prime}\right)$ by Assumption 2.2. In the following lemmas, Assumptions 2.3 and 2.2 are tacitly assumed, and we set $\langle x\rangle:=\left(1+x^{2}\right)^{1 / 2}$ for any $x \in \mathbb{R}^{n}$.

Lemma 3.1. (a) For each $j, k \in\{1, \ldots, d\}$ and each $\gamma, \omega \in \mathbb{C} \backslash \sigma(H)$, the bounded operator $R_{\gamma} H_{j}^{\prime} R_{\omega}$ belongs to $C^{1}\left(\Phi_{k}\right)$.

(b) For each $j, k \in\{1, \ldots, d\}$ the bounded self-adjoint operator $\langle H\rangle^{-2} H_{j}^{\prime}\langle H\rangle^{-2}$ belongs to $C^{1}\left(\Phi_{k}\right)$.

(c) For each $j, k, \ell \in\{1, \ldots, d\}$, the bounded self-adjoint operator $i\left[\langle H\rangle^{-2} H_{j}^{\prime}\langle H\rangle^{-2}, \Phi_{k}\right]$ belongs to $C^{1}\left(\Phi_{\ell}\right)$.

Proof. Due to Assumption 2.2 one has for each $\varphi \in \mathcal{D}\left(\Phi_{k}\right)$

$$
\begin{aligned}
\left\langle\Phi_{k} \varphi, R_{\gamma} H_{j}^{\prime} R_{\omega} \varphi\right\rangle-\left\langle R_{\bar{\omega}} H_{j}^{\prime} R_{\bar{\gamma}} \varphi, \Phi_{k} \varphi\right\rangle \\
=\left\langle\Phi_{k} \varphi, R_{\gamma} H_{j}^{\prime} R_{\omega} \varphi\right\rangle-\left\langle\Phi_{k} R_{\bar{\gamma}} \varphi, H_{j}^{\prime} R_{\omega} \varphi\right\rangle+\left\langle\Phi_{k} R_{\bar{\gamma}} \varphi, H_{j}^{\prime} R_{\omega} \varphi\right\rangle-\left\langle R_{\bar{\omega}} H_{j}^{\prime} R_{\bar{\gamma}} \varphi, \Phi_{k} \varphi\right\rangle \\
=\left\langle\left[R_{\bar{\gamma}}, \Phi_{k}\right] \varphi, H_{j}^{\prime} R_{\omega} \varphi\right\rangle+\left\langle\Phi_{k} R_{\bar{\gamma}} \varphi, H_{j}^{\prime} R_{\omega} \varphi\right\rangle-\left\langle H_{j}^{\prime} R_{\bar{\gamma}} \varphi, \Phi_{k} R_{\omega} \varphi\right\rangle \\
\quad+\left\langle H_{j}^{\prime} R_{\bar{\gamma}} \varphi, \Phi_{k} R_{\omega} \varphi\right\rangle-\left\langle R_{\bar{\omega}} H_{j}^{\prime} R_{\bar{\gamma}} \varphi, \Phi_{k} \varphi\right\rangle \\
=\left\langle\left[R_{\bar{\gamma}}, \Phi_{k}\right] \varphi, H_{j}^{\prime} R_{\omega} \varphi\right\rangle+\left\langle\left[H_{j}^{\prime}, \Phi_{k}\right] R_{\bar{\gamma}} \varphi, R_{\omega} \varphi\right\rangle+\left\langle H_{j}^{\prime} R_{\bar{\gamma}} \varphi,\left[\Phi_{k}, R_{\omega}\right] \varphi\right\rangle .
\end{aligned}
$$

This implies that there exists $\mathrm{C}<\infty$ such that

$$
\left|\left\langle\Phi_{k} \varphi, R_{\gamma} H_{j}^{\prime} R_{\omega} \varphi\right\rangle-\left\langle R_{\bar{\omega}} H_{j}^{\prime} R_{\bar{\gamma}} \varphi, \Phi_{k} \varphi\right\rangle\right| \leq \mathrm{C}\|\varphi\|^{2} .
$$

for each $\varphi \in \mathcal{D}\left(\Phi_{k}\right)$, and thus the first statement follows from [1, Lem. 6.2.9].

For the second statement, since $\langle H\rangle^{-2}=R_{-i} R_{i}$, the operator $\langle H\rangle^{-2} H_{j}^{\prime}\langle H\rangle^{-2}$ is clearly bounded and self-adjoint. Furthermore, by observing that

$$
\langle H\rangle^{-2} H_{j}^{\prime}\langle H\rangle^{-2}=R_{i}\left(R_{-i} H_{j}^{\prime} R_{i}\right) R_{-i}
$$

one concludes from (a) that $\langle H\rangle^{-2} H_{j}^{\prime}\langle H\rangle^{-2}$ is the product of three operators belonging to $C^{1}\left(\Phi_{k}\right)$, and thus belongs to $C^{1}\left(\Phi_{k}\right)$ due to [1, Prop. 5.1.5].

For the last statement, one gets by taking Lemma 2.4 into account

$$
i\left[\langle H\rangle^{-2} H_{j}^{\prime}\langle H\rangle^{-2}, \Phi_{k}\right]=-2\left(R_{i} H_{k}^{\prime} R_{i}\right)\left(R_{-i} H_{j}^{\prime} R_{-i}\right)\left(R_{i}+R_{-i}\right)+\langle H\rangle^{-2} H_{j k}^{\prime \prime}\langle H\rangle^{-2} .
$$

The first term is a product of operators which belong to $C^{1}\left(\Phi_{\ell}\right)$, and thus it belongs to $C^{1}\left(\Phi_{\ell}\right)$. For the second term, a calculation similar to the one presented for the statement (a) using Assumption 2.2 shows that this term also belongs to $C^{1}\left(\Phi_{\ell}\right)$, and so the claim is proved. 
We can now give a precise definition of the conjugate operator $A$ we will use, and prove its self-adjointness. For that purpose, we consider the family

$$
\Pi_{j}:=\langle H\rangle^{-2} H_{j}^{\prime}\langle H\rangle^{-2}, \quad j=1, \ldots, d,
$$

of mutually commuting bounded self-adjoint operators, and we write $\Pi:=\left(\Pi_{1}, \ldots, \Pi_{d}\right)$ for the associated vector operator. Due to Lemma 3.1.(b), each operator $\Pi_{j}$ belongs to $C^{1}\left(\Phi_{k}\right)$. Therefore the operator

$$
A:=\frac{1}{2}(\Pi \cdot \Phi+\Phi \cdot \Pi)
$$

is well-defined and symmetric on $\bigcap_{j=1}^{d} \mathcal{D}\left(\Phi_{j}\right)$. For the next lemma, we note that this set contains the domain $\mathcal{D}\left(\Phi^{2}\right)$ of $\Phi^{2}$.

Lemma 3.2. The operator $A$ is essentially self-adjoint on $\mathcal{D}\left(\Phi^{2}\right)$.

Proof. We use the criterion of essential self-adjointness [27, Thm. X.37].

Given $a>1$, we define the self-adjoint operator $N:=\Phi^{2}+\Pi^{2}+a$ with domain $\mathcal{D}(N) \equiv \mathcal{D}\left(\Phi^{2}\right)$ and observe that in the form sense on $\mathcal{D}(N)$ one has

$$
\begin{aligned}
N^{2} & =\Phi^{4}+\Pi^{4}+a^{2}+2 a \Phi^{2}+2 a \Pi^{2}+\Phi^{2} \Pi^{2}+\Pi^{2} \Phi^{2} \\
& =\Phi^{4}+\Pi^{4}+a^{2}+2 a \Phi^{2}+2 a \Pi^{2}+\sum_{j, k}\left\{\Phi_{j} \Pi_{k}^{2} \Phi_{j}+\Pi_{k} \Phi_{j}^{2} \Pi_{k}\right\}+R
\end{aligned}
$$

with $R:=\sum_{j, k}\left\{\Pi_{k}\left[\Pi_{k}, \Phi_{j}\right] \Phi_{j}+\Phi_{j}\left[\Phi_{j}, \Pi_{k}\right] \Pi_{k}+\left[\Pi_{k}, \Phi_{j}\right]^{2}\right\}$. Now, the following inequality holds

$$
\sum_{j, k}\left\{\Pi_{k}\left[\Pi_{k}, \Phi_{j}\right] \Phi_{j}+\Phi_{j}\left[\Phi_{j}, \Pi_{k}\right] \Pi_{k}\right\} \geq-d \Phi^{2}-\sum_{j, k}\left|\Pi_{k}\left[\Pi_{k}, \Phi_{j}\right]\right|^{2} .
$$

Thus there exists $c>0$ such that $R \geq-d \Phi^{2}-c$. Altogether, we have shown that in the form sense on $\mathcal{D}(N)$

$$
N^{2} \geq \Phi^{4}+\Pi^{4}+\left(a^{2}-c\right)+(2 a-d) \Phi^{2}+2 a \Pi^{2}+\sum_{j, k}\left\{\Phi_{j} \Pi_{k}^{2} \Phi_{j}+\Pi_{k} \Phi_{j}^{2} \Pi_{k}\right\},
$$

where the r.h.s. is a sum of positive terms for $a$ large enough. In particular, one has for $\varphi \in \mathcal{D}(N)$

$$
\|N \varphi\|^{2} \geq\left\|\Pi_{j} \Phi_{j} \varphi\right\|^{2}+\left\|\Phi_{j} \Pi_{j} \varphi\right\|^{2}
$$

which implies that

$$
\|A \varphi\| \leq \frac{1}{2} \sum_{j}\left\{\left\|\Pi_{j} \Phi_{j} \varphi\right\|+\left\|\Phi_{j} \Pi_{j} \varphi\right\|\right\} \leq d\|N \varphi\| .
$$

It remains to estimate the commutator $[A, N]$. In the form sense on $\mathcal{D}(N)$, one has

$$
\begin{aligned}
2[A, N]= & \sum_{j, k}\left\{\left[\Pi_{j}, \Phi_{k}\right] \Phi_{j} \Phi_{k}+\Phi_{k}\left[\Pi_{j}, \Phi_{k}\right] \Phi_{j}+\Phi_{j}\left[\Pi_{j}, \Phi_{k}\right] \Phi_{k}+\Phi_{j} \Phi_{k}\left[\Pi_{j}, \Phi_{k}\right]\right. \\
& \left.+\Pi_{j}\left[\Phi_{j}, \Pi_{k}\right] \Pi_{k}+\Pi_{j} \Pi_{k}\left[\Phi_{j}, \Pi_{k}\right]+\left[\Phi_{j}, \Pi_{k}\right] \Pi_{j} \Pi_{k}+\Pi_{k}\left[\Phi_{j}, \Pi_{k}\right] \Pi_{j}\right\} .
\end{aligned}
$$

The last four terms are bounded. For the other terms, Lemma 3.1.(c), together with the bound

$$
\left|\left\langle\Phi_{j} \varphi, B \Phi_{k}\right\rangle\right| \leq\|B\|\left\langle\varphi, \Phi^{2} \varphi\right\rangle \leq\|B\|\langle\varphi, N \varphi\rangle, \quad \varphi \in \mathcal{D}(N), B \in \mathscr{B}(\mathcal{H}),
$$

leads to the desired estimate, i.e. $\langle\varphi,[A, N] \varphi\rangle \leq$ Const. $\langle\varphi, N \varphi\rangle$.

Lemma 3.3. The operator $H$ is of class $C^{2}(A)$ and the sesquilinear form $i[H, A]$ on $\mathcal{D}(H)$ extends to the bounded positive operator $\langle H\rangle^{-2}\left(H^{\prime}\right)^{2}\langle H\rangle^{-2}$. 
Proof. One has for each $\varphi \in \mathcal{D}\left(\Phi^{2}\right)$ and each $\omega \in \mathbb{C} \backslash \sigma(H)$

$$
\begin{aligned}
2\left\{\left\langle R_{\bar{\omega}} \varphi, A \varphi\right\rangle-\left\langle A \varphi, R_{\omega} \varphi\right\rangle\right\} & =\sum_{j}\left\{\left\langle R_{\bar{\omega}} \varphi,\left(\Pi_{j} \Phi_{j}+\Phi_{j} \Pi_{j}\right) \varphi\right\rangle-\left\langle\left(\Pi_{j} \Phi_{j}+\Phi_{j} \Pi_{j}\right) \varphi, R_{\omega} \varphi\right\rangle\right\} \\
& =\sum_{j}\left\{\left\langle\Pi_{j} \varphi,\left[R_{\omega}, \Phi_{j}\right] \varphi\right\rangle+\left\langle\left[\Phi_{j}, R_{\bar{\omega}}\right] \varphi, \Pi_{j} \varphi\right\rangle\right\} .
\end{aligned}
$$

Since all operators in the last equality are bounded and since $\mathcal{D}\left(\Phi^{2}\right)$ is a core for $A$, this implies that $H$ is of class $C^{1}(A)$ [1, Lem. 6.2.9].

Now observe that the following equalities hold on $\mathcal{H}$

$$
i\left[R_{\omega}, A\right]=\frac{i}{2} \sum_{j}\left\{\Pi_{j}\left[R_{\omega}, \Phi_{j}\right]+\left[R_{\omega}, \Phi_{j}\right] \Pi_{j}\right\}=-R_{\omega}\langle H\rangle^{-2}\left(H^{\prime}\right)^{2}\langle H\rangle^{-2} R_{\omega}
$$

Therefore the sesquilinear form $i[H, A]$ on $\mathcal{D}(H)$ extends to the bounded positive operator $\langle H\rangle^{-2}\left(H^{\prime}\right)^{2}\langle H\rangle^{-2}$. Finally, the operator $i\left[R_{\omega}, A\right]$ can be written as a product of factors in $C^{1}\left(\Phi_{\ell}\right)$ for each $\ell$, namely

$$
i\left[R_{\omega}, A\right]=-\sum_{j} R_{\omega}\left(R_{-i} H_{j}^{\prime} R_{i}\right)\left(R_{-i} H_{j}^{\prime} R_{i}\right) R_{\omega} .
$$

So $i\left[R_{\omega}, A\right]$ also belongs to $C^{1}\left(\Phi_{\ell}\right)$ for each $\ell$, and thus a calculation similar to the one of (3.1) shows that $i\left[R_{\omega}, A\right]$ belongs to $C^{1}(A)$. This implies that $H$ is of class $C^{2}(A)$.

Definition 3.4. A number $\lambda \in \mathbb{R}$ is called a $A$-regular value of $H$ if there exist numbers $a, \delta>0$ such that $\left(H^{\prime}\right)^{2} E^{H}(\lambda ; \delta) \geq a E^{H}(\lambda ; \delta)$. The complement of this set in $\mathbb{R}$ is denoted by $\kappa^{A}(H)$.

The set of $A$-regular values corresponds to the Mourre set with respect to $A$. Indeed, if $\lambda$ is a $A$-regular value, then $\left(H^{\prime}\right)^{2} E^{H}(\lambda ; \delta) \geq a E^{H}(\lambda ; \delta)$ for some $a, \delta>0$ and

$$
E^{H}(\lambda ; \delta) i[H, A] E^{H}(\lambda ; \delta)=E^{H}(\lambda ; \delta)\langle H\rangle^{-2}\left(H^{\prime}\right)^{2}\langle H\rangle^{-2} E^{H}(\lambda ; \delta) \geq a^{\prime} E^{H}(\lambda ; \delta),
$$

where $a^{\prime}:=a \cdot \inf _{\mu \in(\lambda-\delta, \lambda+\delta)}\langle\mu\rangle^{-4}$. In the framework of Mourre theory, this means that the operator $A$ is strictly conjugate to $H$ at the point $\lambda$ [1, Sec. 7.2.2].

Lemma 3.5. The sets $\kappa(H)$ and $\kappa^{A}(H)$ are equal.

Proof. Let $\lambda$ be a $A$-regular value of $H$. Then there exist $a, \delta>0$ such that

$$
E^{H}(\lambda ; \delta) \leq a^{-1}\left(H^{\prime}\right)^{2} E^{H}(\lambda ; \delta),
$$

and we obtain for $\varepsilon>0$ :

$$
\begin{aligned}
\left\|\left[\left(H^{\prime}\right)^{2}+\varepsilon\right]^{-1} E^{H}(\lambda ; \delta)\right\|^{2} & =\sup _{\varphi \in \mathcal{H},\|\varphi\|=1}\left\langle\left[\left(H^{\prime}\right)^{2}+\varepsilon\right]^{-1} \varphi, E^{H}(\lambda ; \delta)\left[\left(H^{\prime}\right)^{2}+\varepsilon^{2}\right]^{-1} \varphi\right\rangle \\
& \leq a^{-2} \sup _{\varphi \in \mathcal{H},\|\varphi\|=1}\left\langle\left[\left(H^{\prime}\right)^{2}+\varepsilon\right]^{-1} \varphi, E^{H}(\lambda ; \delta)\left(H^{\prime}\right)^{4}\left[\left(H^{\prime}\right)^{2}+\varepsilon\right]^{-1} \varphi\right\rangle \\
& \leq a^{-2}\left\|\left(H^{\prime}\right)^{2}\left[\left(H^{\prime}\right)^{2}+\varepsilon\right]^{-1}\right\|^{2} \\
& \leq a^{-2}
\end{aligned}
$$

which implies, by taking the $\operatorname{limit} \lim _{\varepsilon \searrow 0}$, that $\lambda$ is a regular value.

Now, let $\lambda$ be a regular value of $H$. Then there exists $\delta>0$ such that

$$
\begin{aligned}
\text { Const. } \geq \lim _{\varepsilon \searrow 0}\left\|\left[\left(H^{\prime}\right)^{2}+\varepsilon\right]^{-1} E^{H}(\lambda ; \delta)\right\| & =\lim _{\varepsilon \searrow 0}\left\|E^{H}(\lambda ; \delta)\left[\left(H^{\prime}\right)^{2} E^{H}(\lambda ; \delta)+\varepsilon\right]^{-1} E^{H}(\lambda ; \delta)\right\| \\
& =\lim _{\varepsilon \searrow 0}\left\|\left[\left(H^{\prime}\right)^{2} E^{H}(\lambda ; \delta)+\varepsilon\right]^{-1}\right\|_{\mathscr{B}\left(\mathcal{H}_{\lambda, \delta}\right)},
\end{aligned}
$$


where $\mathcal{H}_{\lambda, \delta}:=E^{H}(\lambda ; \delta) \mathcal{H}$. But we have

$$
\left\|\left[\left(H^{\prime}\right)^{2} E^{H}(\lambda ; \delta)+\varepsilon\right]^{-1}\right\|_{\mathscr{B}\left(\mathcal{H}_{\lambda, \delta}\right)}=(a+\varepsilon)^{-1},
$$

where the number $a \geq 0$ is the infimum of the spectrum of $\left(H^{\prime}\right)^{2} E^{H}(\lambda ; \delta)$, considered as an operator in $\mathcal{H}_{\lambda, \delta}$. Therefore, Formula (3.2) entails the bound $a^{-1} \leq$ Const., which implies that $a>0$. In consequence, the operator $\left(H^{\prime}\right)^{2} E^{H}(\lambda ; \delta)$ is strictly positive in $\mathcal{H}_{\lambda, \delta}$, namely,

$$
\left(H^{\prime}\right)^{2} E^{H}(\lambda ; \delta) \geq a E^{H}(\lambda ; \delta)
$$

with $a>0$. This implies that $\lambda$ is a $A$-regular value of $H$, and $\kappa(H)$ is equal to $\kappa^{A}(H)$.

We shall now state our main result on the nature of the spectrum of $H$, and exhibit a class of locally $H$ smooth operators. The space $(\mathcal{D}(A), \mathcal{H})_{1 / 2,1}$, defined by real interpolation [1, Sec. 3.4.1], is denoted by $\mathscr{K}$. Since for each $j \in\{1, \ldots, d\}$ the operator $\Pi_{j}$ belongs to $C^{1}\left(\Phi_{j}\right)$, we have $\mathcal{D}(\langle\Phi\rangle) \subset \mathcal{D}(A)$, and it follows from [1, Thm. 2.6.3] and [1, Thm. 3.4.3.(a)] that for $s>1 / 2$ the continuous embeddings hold:

$$
\mathcal{D}\left(\langle\Phi\rangle^{s}\right) \subset \mathscr{K} \subset \mathcal{H} \subset \mathscr{K}^{*} \subset \mathcal{D}\left(\langle\Phi\rangle^{-s}\right) .
$$

The symbol $\mathbb{C}_{ \pm}$stands for the half-plane $\mathbb{C}_{ \pm}:=\{\omega \in \mathbb{C} \mid \pm \operatorname{Im}(\omega)>0\}$.

Theorem 3.6. Let $H$ satisfy Assumptions 2.2 and 2.3. Then,

(a) the spectrum of $H$ in $\sigma(H) \backslash \kappa(H)$ is purely absolutely continuous,

(b) each operator $T \in \mathscr{B}\left(\mathcal{D}\left(\langle\Phi\rangle^{-s}\right), \mathcal{H}\right)$, with $s>1 / 2$, is locally $H$-smooth on $\mathbb{R} \backslash \kappa(H)$.

Proof. (a) This is a direct application of [31, Thm. 0.1] which takes Lemmas 3.3 and 3.5 into account.

(b) We know from [31, Thm. 0.1] that the map $\omega \mapsto R_{\omega} \in \mathscr{B}\left(\mathscr{K}, \mathscr{K}^{*}\right)$, which is holomorphic on the halfplane $\mathbb{C}_{ \pm}$, extends to a weak ${ }^{*}$-continuous function on $\mathbb{C}_{ \pm} \cup\{\mathbb{R} \backslash \kappa(H)\}$. Now, consider $T \in \mathscr{B}\left(\mathscr{K}^{*}, \mathcal{H}\right)$. Then one has $T^{*} \in \mathscr{B}(\mathcal{H}, \mathscr{K})$, and it follows from the above continuity that for each compact subset $J \subset \mathbb{R} \backslash \kappa(H)$ there exists a constant $\mathrm{C} \geq 0$ such that for all $\omega \in \mathbb{C}$ with $\operatorname{Re}(\omega) \in J$ and $\operatorname{Im}(\omega) \in(0,1)$ one has

$$
\left\|T R_{\omega} T^{*}\right\|+\left\|T R_{\bar{\omega}} T^{*}\right\| \leq \mathrm{C} .
$$

A fortiori, one also has $\sup _{\omega}\left\|T\left(R_{\omega}-R_{\bar{\omega}}\right) T^{*}\right\| \leq \mathrm{C}$, where the supremum is taken over the same set of complex numbers. This last property is equivalent to the local $H$-smoothness of $T$ on $\mathbb{R} \backslash \kappa(H)$. The claim is then obtained by using the last embedding of (3.3).

\section{Averaged localisation functions}

In this section we recall some properties of a class of averaged localisation functions which appears naturally when dealing with quantum scattering theory. These functions, which are denoted $R_{f}$, are constructed in terms of functions $f \in \mathrm{L}^{\infty}\left(\mathbb{R}^{d}\right)$ of localisation around the origin 0 of $\mathbb{R}^{d}$. They were already used, in one form or another, in [15], [36], and [37].

Assumption 4.1. The function $f \in \mathrm{L}^{\infty}\left(\mathbb{R}^{d}\right)$ satisfies the following conditions:

(i) There exists $\rho>0$ such that $|f(x)| \leq$ Const. $\langle x\rangle^{-\rho}$ for a.e. $x \in \mathbb{R}^{d}$.

(ii) $f=1$ on a neighbourhood of 0 .

It is clear that $\mathrm{s}-\lim _{r \rightarrow \infty} f(\Phi / r)=1$ if $f$ satisfies Assumption 4.1. Furthermore, one has for each $x \in$ $\mathbb{R}^{d} \backslash\{0\}$

$$
\left|\int_{0}^{\infty} \frac{\mathrm{d} \mu}{\mu}\left[f(\mu x)-\chi_{[0,1]}(\mu)\right]\right| \leq \int_{0}^{1} \frac{\mathrm{d} \mu}{\mu}|f(\mu x)-1|+\text { Const. } \int_{1}^{+\infty} \mathrm{d} \mu \mu^{-(1+\rho)}<\infty,
$$


where $\chi_{[0,1]}$ denotes the characteristic function for the interval $[0,1]$. Therefore the function $R_{f}: \mathbb{R}^{d} \backslash\{0\} \rightarrow \mathbb{C}$ given by

$$
R_{f}(x):=\int_{0}^{+\infty} \frac{\mathrm{d} \mu}{\mu}\left[f(\mu x)-\chi_{[0,1]}(\mu)\right]
$$

is well-defined. If $\mathbb{R}_{+}^{*}:=(0, \infty)$, endowed with the multiplication, is seen as a Lie group with Haar measure $\frac{\mathrm{d} \mu}{\mu}$, then $R_{f}$ is the renormalised average of $f$ with respect to the (dilation) action of $\mathbb{R}_{+}^{*}$ on $\mathbb{R}^{d}$.

In the next lemma we recall some differentiability and homogeneity properties of $R_{f}$. We also give the explicit form of $R_{f}$ when $f$ is a radial function. The reader is referred to [37, Sec. 2] for proofs and details. The symbol $\mathscr{S}\left(\mathbb{R}^{d}\right)$ stands for the Schwartz space on $\mathbb{R}^{d}$.

Lemma 4.2. Let $f$ satisfy Assumption 4.1.

(a) Assume that $\left(\partial_{j} f\right)(x)$ exists for all $j \in\{1, \ldots, d\}$ and $x \in \mathbb{R}^{d}$, and suppose that there exists some $\rho>0$ such that $\left|\left(\partial_{j} f\right)(x)\right| \leq$ Const. $\langle x\rangle^{-(1+\rho)}$ for each $x \in \mathbb{R}^{d}$. Then $R_{f}$ is differentiable on $\mathbb{R}^{d} \backslash\{0\}$, and its derivative is given by

$$
R_{f}^{\prime}(x)=\int_{0}^{\infty} \mathrm{d} \mu f^{\prime}(\mu x) .
$$

In particular, if $f \in \mathscr{S}\left(\mathbb{R}^{d}\right)$ then $R_{f}$ belongs to $C^{\infty}\left(\mathbb{R}^{d} \backslash\{0\}\right)$.

(b) Assume that $R_{f}$ belongs to $C^{m}\left(\mathbb{R}^{d} \backslash\{0\}\right)$ for some $m \geq 1$. Then one has for each $x \in \mathbb{R}^{d} \backslash\{0\}$ and $t>0$ the homogeneity properties

$$
\begin{aligned}
x \cdot R_{f}^{\prime}(x) & =-1, \\
t^{|\alpha|}\left(\partial^{\alpha} R_{f}\right)(t x) & =\left(\partial^{\alpha} R_{f}\right)(x),
\end{aligned}
$$

where $\alpha \in \mathbb{N}^{d}$ is a multi-index with $1 \leq|\alpha| \leq m$.

(c) Assume that $f$ is radial, i.e. there exists $f_{0} \in \mathrm{L}^{\infty}(\mathbb{R})$ such that $f(x)=f_{0}(|x|)$ for a.e. $x \in \mathbb{R}^{d}$. Then $R_{f}$ belongs to $C^{\infty}\left(\mathbb{R}^{d} \backslash\{0\}\right)$, and $R_{f}^{\prime}(x)=-x^{-2} x$.

Obviously, one can show as in Lemma 4.2.(a) that $R_{f}$ is of class $C^{m}\left(\mathbb{R}^{d} \backslash\{0\}\right)$ if one has for each $\alpha \in \mathbb{N}^{d}$ with $|\alpha| \leq m$ that $\left(\partial^{\alpha} f\right)(x)$ exists and that $\left|\left(\partial^{\alpha} f\right)(x)\right| \leq$ Const. $\langle x\rangle^{-(|\alpha|+\rho)}$ for some $\rho>0$. However, this is not a necessary condition. In some cases (as in Lemma 4.2.(c)), the function $R_{f}$ is very regular outside the point 0 even if $f$ is not continuous.

\section{Integral formula}

In this section we prove our main result on the relation between the evolution of the localisation operators $f(\Phi / r)$ and the time operator $T_{f}$ defined below. We begin with a technical lemma that will be used subsequently. Since this result could also be useful in other situations, we present here a general version of it. The symbol $\mathscr{F}$ stands for the Fourier transformation, and the measure $\underline{\mathrm{d}} x$ on $\mathbb{R}^{n}$ is chosen so that $\mathscr{F}$ extends to a unitary operator in $\mathrm{L}^{2}\left(\mathbb{R}^{n}\right)$.

Proposition 5.1. Let $C \equiv\left(C_{1}, \ldots, C_{n}\right)$ and $D \equiv\left(D_{1}, \ldots, D_{d}\right)$ be two families of mutually commuting selfadjoint operators in a Hilbert space $\mathscr{H}$. Let $k \geq 1$ be an integer, and assume that each $C_{j}$ is of class $C^{k}(D)$. Let $f \in \mathrm{L}^{\infty}\left(\mathbb{R}^{n}\right)$, set $g(x):=f(x)\left\langle x_{1}\right\rangle^{2 k} \cdots\left\langle x_{n}\right\rangle^{2 k}$, and suppose that the functions $g$ and

$$
x \mapsto(\mathscr{F} g)(x)\left\langle x_{1}\right\rangle^{k+1} \cdots\left\langle x_{n}\right\rangle^{k+1}
$$

are in $\mathrm{L}^{1}\left(\mathbb{R}^{n}\right)$. Then the operator $f(C)$ belongs to $C^{k}(D)$. In particular, if $f \in \mathscr{S}\left(\mathbb{R}^{n}\right)$ then $f(C)$ belongs to $C^{k}(D)$. 
Proof. For each $y \in \mathbb{R}^{d}$, we set $D_{y}:=\frac{1}{i|y|}\left(\mathrm{e}^{i y \cdot D}-1\right)$. Then we know from [1, Lemma 6.2.3.(a)] that it is sufficient to prove that $\left\|\operatorname{ad}_{D_{y}}^{k}(f(C))\right\|$ is bounded by a constant independent of $y$. By using the linearity of $\operatorname{ad}_{D_{y}}^{k}(\cdot)$ and [1, Eq. 5.1.16], we get

$$
\begin{aligned}
& \operatorname{ad}_{D_{y}}^{k}(f(C)) \\
& =\operatorname{ad}_{D_{y}}^{k}\left(g(C)\left\langle C_{1}\right\rangle^{-2 k} \cdots\left\langle C_{n}\right\rangle^{-2 k}\right) \\
& =\int_{\mathbb{R}^{n}} \underline{\mathrm{d}} x(\mathscr{F} g)(x) \operatorname{ad}_{D_{y}}^{k}\left(\mathrm{e}^{i x_{1} C_{1}}\left\langle C_{1}\right\rangle^{-2 k} \cdots \mathrm{e}^{i x_{2} C_{n}}\left\langle C_{n}\right\rangle^{-2 k}\right) \\
& =\sum_{k_{1}+\cdots+k_{n}=k} \mathrm{C}_{k_{1} \cdots k_{n}} \int_{\mathbb{R}^{n}} \underline{\mathrm{d}} x(\mathscr{F} g)(x) \operatorname{ad}_{D_{y}}^{k_{1}}\left(\mathrm{e}^{i x_{1} C_{1}}\left\langle C_{1}\right\rangle^{-2 k}\right) \cdots \operatorname{ad}_{D_{y}}^{k_{n}}\left(\mathrm{e}^{i x_{2} C_{n}}\left\langle C_{n}\right\rangle^{-2 k}\right),
\end{aligned}
$$

where $\mathrm{C}_{k_{1} \cdots k_{n}}>0$ is some explicit constant. Furthermore, since $C_{j}$ is of class $C^{k}(D)$, we know from [1, Eq. 6.2.13] that

$$
\left\|\operatorname{ad}_{G_{y}}^{k_{j}}\left(\mathrm{e}^{i x_{j} C_{j}}\left\langle C_{j}\right\rangle^{-2 k}\right)\right\| \leq \mathrm{C}_{k_{j}}\left\langle x_{j}\right\rangle^{k+1},
$$

where $\mathrm{C}_{k_{j}} \geq 0$ is independent of $y$ and $x_{j}$. This implies that

$$
\left\|\operatorname{ad}_{D_{y}}^{k}(f(C))\right\| \leq \text { Const. } \int_{\mathbb{R}^{n}} \underline{\mathrm{d}} x|(\mathscr{F} g)(x)|\left\langle x_{1}\right\rangle^{k+1} \cdots\left\langle x_{n}\right\rangle^{k+1} \leq \text { Const. },
$$

and the claim is proved.

In Lemma 2.6.(a) we have shown that the set $\kappa(H)$ is closed. So we can define for each $t \geq 0$ the set

$$
\mathscr{D}_{t}:=\left\{\varphi \in \mathcal{D}\left(\langle\Phi\rangle^{t}\right) \mid \varphi=\eta(H) \varphi \text { for some } \eta \in C_{\mathrm{c}}^{\infty}(\mathbb{R} \backslash \kappa(H))\right\} .
$$

The set $\mathscr{D}_{t}$ is included in the subspace $\mathcal{H}_{\mathrm{ac}}(H)$ of absolute continuity of $H$, due to Theorem 3.6, and $\mathscr{D}_{t_{1}} \subset \mathscr{D}_{t_{2}}$ if $t_{1} \geq t_{2}$. We refer the reader to Section 6 for an account on density properties of the sets $\mathscr{D}_{t}$.

In the sequel we consider the set of operators $\left\{H_{j k}^{\prime \prime}\right\}$ as the components of a $d$-dimensional (Hessian) matrix which we denote by $H^{\prime \prime}$. Furthermore we shall sometimes write $C^{-1}$ for an operator $C$ a priori not invertible. In such a case, the operator $C^{-1}$ will always be restricted to a set where it is well-defined. Namely, if $\mathcal{D}$ is a set on which $C$ is invertible, then we shall simply write " $C^{-1}$ acting on $\mathcal{D}$ " instead of using the notation $\left.C^{-1}\right|_{\mathcal{D}}$.

Proposition 5.2. Let $H$ and $\Phi$ satisfy Assumptions 2.2 and 2.3. Let $f$ satisfy Assumption 4.1 and assume that $R_{f}$ belongs to $C^{1}\left(\mathbb{R}^{d} \backslash\{0\}\right)$. Then the map

$$
t_{f}: \mathscr{D}_{1} \rightarrow \mathbb{C}, \quad \varphi \mapsto t_{f}(\varphi):=-\frac{1}{2} \sum_{j}\left\{\left\langle\Phi_{j} \varphi,\left(\partial_{j} R_{f}\right)\left(H^{\prime}\right) \varphi\right\rangle+\left\langle\left(\partial_{j} R_{\bar{f}}\right)\left(H^{\prime}\right) \varphi, \Phi_{j} \varphi\right\rangle\right\},
$$

is well-defined. Moreover, if $\left(\partial_{j} R_{f}\right)\left(H^{\prime}\right) \varphi$ belongs to $\mathcal{D}\left(\Phi_{j}\right)$ for each $j$, then the linear operator $T_{f}: \mathscr{D}_{1} \rightarrow \mathcal{H}$ defined by

$$
T_{f} \varphi:=-\frac{1}{2}\left(\Phi \cdot R_{f}^{\prime}\left(H^{\prime}\right)+R_{f}^{\prime}\left(\frac{H^{\prime}}{\left|H^{\prime}\right|}\right) \cdot \Phi\left|H^{\prime}\right|^{-1}+i R_{f}^{\prime}\left(\frac{H^{\prime}}{\left|H^{\prime}\right|}\right) \cdot\left(H^{\prime \prime} H^{\prime}\right)\left|H^{\prime}\right|^{-3}\right) \varphi
$$

satisfies $t_{f}(\varphi)=\left\langle v, T_{f} \varphi\right\rangle$ for each $\varphi \in \mathscr{D}_{1}$. In particular, $T_{f}$ is a symmetric operator if $f$ is real and if $\mathscr{D}_{1}$ is dense in $\mathcal{H}$.

Remark 5.3. Formula (5.1) is a priori rather complicated and one could be tempted to replace it by the simpler formula $-\frac{1}{2}\left(\Phi \cdot R_{f}^{\prime}\left(H^{\prime}\right)+R_{f}^{\prime}\left(H^{\prime}\right) \cdot \Phi\right)$. Unfortunately, a precise meaning of this expression is not available in general, and its full derivation can only be justified in concrete examples. 
Remark 5.4. If $\varphi \in \mathscr{D}_{1}$ and if $f$ either belongs to $\mathscr{S}\left(\mathbb{R}^{d}\right)$ or is radial, then the assumption $\left(\partial_{j} R_{f}\right)\left(H^{\prime}\right) \varphi \in$ $\mathcal{D}\left(\Phi_{j}\right)$ holds for each $j$. Indeed, by Lemma 2.6.(d) there exists $\eta \in C_{\mathrm{c}}^{\infty}((0, \infty))$ such that $\left(\partial_{j} R_{f}\right)\left(H^{\prime}\right) \varphi=$ $\left(\partial_{j} R_{f}\right)\left(H^{\prime}\right) \eta\left(\left(H^{\prime}\right)^{2}\right) \varphi$. By Lemma 4.2 and Proposition 5.1, it then follows that $\left(\partial_{j} R_{f}\right)\left(H^{\prime}\right) \eta\left(\left(H^{\prime}\right)^{2}\right) \in$ $C^{1}\left(\Phi_{j}\right)$, which implies the statement.

Proof of Proposition 5.2. Let $\varphi \in \mathscr{D}_{1}$. Then Lemma 2.6.(d) implies that there exists a function $\eta \in C_{\mathrm{c}}^{\infty}((0, \infty))$ such that

$$
\left(\partial_{j} R_{f}\right)\left(H^{\prime}\right) \varphi=\left(\partial_{j} R_{f}\right)\left(H^{\prime}\right) \eta\left(\left(H^{\prime}\right)^{2}\right) \varphi .
$$

Thus $\left\|\left(\partial_{j} R_{f}\right)\left(H^{\prime}\right) \varphi\right\| \leq$ Const. $\|\varphi\|$, and we have

$$
\left|t_{f}(\varphi)\right| \leq \text { Const. }\|\varphi\| \cdot\|\langle\Phi\rangle \varphi\|,
$$

which implies the first part of the claim.

For the second part of the claim, it is sufficient to show that

$$
\begin{aligned}
& \sum_{j}\left\langle\left(\partial_{j} R_{\bar{f}}\right)\left(H^{\prime}\right) \varphi, \Phi_{j} \varphi\right\rangle \\
& =\left\langle\varphi,\left\{R_{f}^{\prime}\left(\frac{H^{\prime}}{\left|H^{\prime}\right|}\right) \cdot \Phi\left|H^{\prime}\right|^{-1}+i R_{f}^{\prime}\left(\frac{H^{\prime}}{\left|H^{\prime}\right|}\right) \cdot\left(H^{\prime \prime} H^{\prime}\right)\left|H^{\prime}\right|^{-3}\right\} \varphi\right\rangle .
\end{aligned}
$$

Using Formula (4.2), Lemma 2.6.(d), and [10, Eq. 4.3.2], one gets

$$
\begin{aligned}
& \sum_{j}\left\langle\left(\partial_{j} R_{\bar{f}}\right)\left(H^{\prime}\right) \varphi, \Phi_{j} \varphi\right\rangle \\
& =\sum_{j}\left\langle\left(\partial_{j} R_{\bar{f}}\right)\left(\frac{H^{\prime}}{\left|H^{\prime}\right|}\right)\left|H^{\prime}\right|^{-1} \varphi, \Phi_{j} \varphi\right\rangle \\
& =\sum_{j} \lim _{\varepsilon \searrow 0}\left\langle\left(\partial_{j} R_{\bar{f}}\right)\left(\frac{H^{\prime}}{\left|H^{\prime}\right|}\right) \varphi,\left[\left(H^{\prime}\right)^{2}+\varepsilon\right]^{-1 / 2} \Phi_{j} \varphi\right\rangle \\
& =\left\langle\varphi, R_{f}^{\prime}\left(\frac{H^{\prime}}{\left|H^{\prime}\right|}\right) \cdot \Phi\left|H^{\prime}\right|^{-1} \varphi\right\rangle \\
& \quad+\pi^{-1} \sum_{j} \lim _{\varepsilon \searrow 0} \int_{0}^{\infty} \mathrm{d} t t^{-1 / 2}\left\langle\left(\partial_{j} R_{\bar{f}}\right)\left(\frac{H^{\prime}}{\left|H^{\prime}\right|}\right) \varphi,\left[\left[\left(H^{\prime}\right)^{2}+\varepsilon+t\right]^{-1}, \Phi_{j}\right] \varphi\right\rangle .
\end{aligned}
$$

Now, by using Assumption 2.2 and Lemma 2.4 one obtains that

$$
\left[\left[\left(H^{\prime}\right)^{2}+\varepsilon+t\right]^{-1}, \Phi_{j}\right] \varphi=2 i\left[\left(H^{\prime}\right)^{2}+\varepsilon+t\right]^{-2}\left(H^{\prime \prime} H^{\prime}\right)_{j} \varphi
$$

It follows that

$$
\begin{aligned}
& \pi^{-1} \sum_{j} \lim _{\varepsilon \backslash 0} \int_{0}^{\infty} \mathrm{d} t t^{-1 / 2}\left\langle\left(\partial_{j} R_{\bar{f}}\right)\left(\frac{H^{\prime}}{\left|H^{\prime}\right|}\right) \varphi, 2 i\left[\left(H^{\prime}\right)^{2}+\varepsilon+t\right]^{-2}\left(H^{\prime \prime} H^{\prime}\right)_{j} \varphi\right\rangle \\
& =\sum_{j} \lim _{\varepsilon \searrow 0}\left\langle\left(\partial_{j} R_{\bar{f}}\right)\left(\frac{H^{\prime}}{\left|H^{\prime}\right|}\right) \varphi, i\left[\left(H^{\prime}\right)^{2}+\varepsilon\right]^{-3 / 2}\left(H^{\prime \prime} H^{\prime}\right)_{j} \varphi\right\rangle \\
& =\left\langle\varphi, i R_{f}^{\prime}\left(\frac{H^{\prime}}{\left|H^{\prime}\right|}\right) \cdot\left(H^{\prime \prime} H^{\prime}\right)\left|H^{\prime}\right|^{-3} \varphi\right\rangle,
\end{aligned}
$$

and thus

$$
\sum_{j}\left\langle\left(\partial_{j} R_{\bar{f}}\right)\left(H^{\prime}\right) \varphi, \Phi_{j} \varphi\right\rangle=\left\langle\varphi,\left\{R_{f}^{\prime}\left(\frac{H^{\prime}}{\left|H^{\prime}\right|}\right) \cdot \Phi\left|H^{\prime}\right|^{-1}+i R_{f}^{\prime}\left(\frac{H^{\prime}}{\left|H^{\prime}\right|}\right) \cdot\left(H^{\prime \prime} H^{\prime}\right)\left|H^{\prime}\right|-3\right\} \varphi\right\rangle .
$$


Suppose for a while that $f$ is radial. Then one has $\left(\partial_{j} R_{f}\right)(x)=-x^{-2} x_{j}$ due to Lemma 4.2.(c), and Formula (5.1) holds by Remark 5.4. This implies that $T_{f}$ is equal to

$$
T:=\frac{1}{2}\left(\Phi \cdot \frac{H^{\prime}}{\left(H^{\prime}\right)^{2}}+\frac{H^{\prime}}{\left|H^{\prime}\right|} \cdot \Phi\left|H^{\prime}\right|^{-1}+\frac{i H^{\prime}}{\left(H^{\prime}\right)^{4}} \cdot\left(H^{\prime \prime} H^{\prime}\right)\right)
$$

on $\mathscr{D}_{1}$.

The next theorem is our main result; it relates the evolution of localisation operators $f(\Phi / r)$ to the operator $T_{f}$. In its proof, we freely use the notations of [1] for some regularity classes with respect to the unitary group generated by $\Phi$. For us, a function $f: \mathbb{R}^{d} \rightarrow \mathbb{C}$ is even if $f(x)=f(-x)$ for a.e. $x \in \mathbb{R}^{d}$.

Theorem 5.5. Let $H$ and $\Phi$ satisfy Assumptions 2.2 and 2.3. Let $f \in \mathscr{S}\left(\mathbb{R}^{d}\right)$ be an even function such that $f=1$ on a neighbourhood of 0 . Then we have for each $\varphi \in \mathscr{D}_{2}$

$$
\lim _{r \rightarrow \infty} \frac{1}{2} \int_{0}^{\infty} \mathrm{d} t\left\langle\varphi,\left[\mathrm{e}^{-i t H} f(\Phi / r) \mathrm{e}^{i t H}-\mathrm{e}^{i t H} f(\Phi / r) \mathrm{e}^{-i t H}\right] \varphi\right\rangle=t_{f}(\varphi) .
$$

Note that the integral on the 1.h.s. of (5.3) is finite for each $r>0$ since $f(\Phi / r)$ can be factorized as

$$
f(\Phi / r) \equiv|f(\Phi / r)|^{1 / 2} \cdot \operatorname{sgn}[f(\Phi / r)] \cdot|f(\Phi / r)|^{1 / 2},
$$

with $|f(\Phi / r)|^{1 / 2}$ locally $H$-smooth on $\mathbb{R} \backslash \kappa(H)$ by Theorem 3.6. Furthermore, since Remark 5.4 applies, the r.h.s. can also be written as the expectation value $\left\langle\varphi, T_{f} \varphi\right\rangle$.

Proof. (i) Let $\varphi \in \mathscr{D}_{2}$, take a real $\eta \in C_{\mathrm{c}}^{\infty}(\mathbb{R} \backslash \kappa(H))$ such that $\eta(H) \varphi=\varphi$, and set $\eta_{t}(H):=\mathrm{e}^{i t H} \eta(H)$. Then we have

$$
\begin{aligned}
& \left\langle\varphi,\left[\mathrm{e}^{i t H} f(\Phi / r) \mathrm{e}^{-i t H}-\mathrm{e}^{-i t H} f(\Phi / r) \mathrm{e}^{i t H}\right] \varphi\right\rangle \\
& =\int_{\mathbb{R}^{d}} \underline{\mathrm{d}} x(\mathscr{F} f)(x)\left\langle\varphi,\left[\eta_{t}(H) \mathrm{e}^{i \frac{x}{r} \cdot \Phi} \eta_{-t}(H)-\eta_{-t}(H) \mathrm{e}^{i \frac{x}{r} \cdot \Phi} \eta_{t}(H)\right] \varphi\right\rangle \\
& =\int_{\mathbb{R}^{d}} \underline{\mathrm{d}} x(\mathscr{F} f)(x)\left\langle\varphi,\left[\mathrm{e}^{i \frac{x}{r} \cdot \Phi} \eta_{t}\left(H\left(\frac{x}{r}\right)\right) \eta_{-t}(H)-\eta_{-t}(H) \eta_{t}\left(H\left(-\frac{x}{r}\right)\right) \mathrm{e}^{i \frac{x}{r} \cdot \Phi}\right] \varphi\right\rangle \\
& =\int_{\mathbb{R}^{d}} \underline{\mathrm{d}} x(\mathscr{F} f)(x)\left\langle\varphi,\left\{\left(\mathrm{e}^{i \frac{x}{r} \cdot \Phi}-1\right) \eta_{t}\left(H\left(\frac{x}{r}\right)\right) \eta_{-t}(H)\right.\right. \\
& \left.\left.\quad+\eta_{-t}(H)\left[\eta_{t}\left(H\left(\frac{x}{r}\right)\right)-\eta_{t}\left(H\left(-\frac{x}{r}\right)\right)\right]-\eta_{-t}(H) \eta_{t}\left(H\left(-\frac{x}{r}\right)\right)\left(\mathrm{e}^{i \frac{x}{r} \cdot \Phi}-1\right)\right\} \varphi\right\rangle .
\end{aligned}
$$

Since $f$ is even, $\mathscr{F} f$ is also even, and

$$
\int_{\mathbb{R}^{d}} \underline{\mathrm{d}} x(\mathscr{F} f)(x)\left\langle\varphi, \eta_{-t}(H)\left[\eta_{t}\left(H\left(\frac{x}{r}\right)\right)-\eta_{t}\left(H\left(-\frac{x}{r}\right)\right)\right] \varphi\right\rangle=0 .
$$

Thus Formula (5.4), Lemma 2.4, and the change of variables $\mu:=t / r, \nu:=1 / r$, give

$$
\lim _{r \rightarrow \infty} \frac{1}{2} \int_{0}^{\infty} \mathrm{d} t\left\langle\varphi,\left[\mathrm{e}^{-i t H} f(\Phi / r) \mathrm{e}^{i t H}-\mathrm{e}^{i t H} f(\Phi / r) \mathrm{e}^{-i t H}\right] \varphi\right\rangle=-\frac{1}{2} \lim _{\nu \searrow 0} \int_{0}^{\infty} \mathrm{d} \mu \int_{\mathbb{R}^{d}} \underline{\mathrm{d}} x K(\nu, \mu, x),
$$

where

$$
\begin{aligned}
K(\nu, \mu, x):=(\mathscr{F} f)(x)\langle\varphi,\{ & \frac{1}{\nu}\left(\mathrm{e}^{i \nu x \cdot \Phi}-1\right) \eta(H(\nu x)) \mathrm{e}^{i \frac{\mu}{\nu}[H(\nu x)-H]} \\
& \left.\left.-\eta(H(-\nu x)) \mathrm{e}^{i \frac{\mu}{\nu}[H(-\nu x)-H]} \frac{1}{\nu}\left(\mathrm{e}^{i \nu x \cdot \Phi}-1\right)\right\} \varphi\right\rangle .
\end{aligned}
$$

(ii) To prove the statement, we shall show that one may interchange the limit and the integrals in (5.5), by invoking Lebesgue's dominated convergence theorem. This will be done in (iii) below. Here we pursue the calculations assuming that these interchanges are justified.

We know from Assumption 2.2 that $H$ is of class $C^{2}\left(\Phi_{j}\right)$ (and thus of class $C^{1,1}\left(\Phi_{j}\right)$ ) for each $j \in$ $\{1, \ldots, d\}$. Since the domain of $H$ is invariant under the group generated by $\Phi_{j}$, it follows then from [1, 
Thm. 6.3.4.(b)] that $H$ belongs to $C^{1,1}\left(\Phi_{j}, \mathcal{G}, \mathcal{G}^{*}\right)$, where $\mathcal{G}$ denotes the space $\mathcal{D}(H)$ endowed with the graph topology. In particular, $H$ belongs to $C_{u}^{1}\left(\Phi_{j}, \mathcal{G}, \mathcal{G}^{*}\right)$; namely, the map $\mathbb{R} \ni \nu \mapsto H\left(\nu e_{j}\right) \in \mathscr{B}\left(\mathcal{G}, \mathcal{G}^{*}\right)$ is continuously differentiable in the uniform topology. Therefore the map

$$
\mathbb{R} \backslash\{0\} \ni \nu \mapsto \frac{1}{\nu}\left[H\left(\nu e_{j}\right)-H\right] \in \mathscr{B}\left(\mathcal{G}, \mathcal{G}^{*}\right)
$$

extends to a continuous map defined on $\mathbb{R}$ and taking value $H_{j}^{\prime}$ at $\nu=0$.

Now, the exponential map $B \mapsto \mathrm{e}^{i B}$ is continuous from $\mathscr{B}\left(\mathcal{G}, \mathcal{G}^{*}\right)$ to $\mathscr{B}\left(\mathcal{G}, \mathcal{G}^{*}\right)$. So, the composed map

$$
\mathbb{R} \ni \nu \mapsto \mathrm{e}^{\frac{i}{\nu}\left[H\left(\nu e_{j}\right)-H\right]} \in \mathscr{B}\left(\mathcal{G}, \mathcal{G}^{*}\right)
$$

is also continuous, and takes value $\mathrm{e}^{i H_{j}^{\prime}}$ at $\nu=0$. By linearity and by taking Lemma 2.4 into account, one finally obtains in $\mathscr{B}\left(\mathcal{G}, \mathcal{G}^{*}\right)$

$$
\lim _{\nu \searrow 0} \mathrm{e}^{i \frac{\mu}{\nu}[H(\nu x)-H]}=\mathrm{e}^{i \mu x \cdot H^{\prime}} .
$$

It follows that for any $\varphi, \psi \in \mathcal{G}$, one has

$$
\lim _{\nu \searrow 0}\left\langle\psi, \mathrm{e}^{i \frac{\mu}{\nu}[H(\nu x)-H]} \varphi\right\rangle=\left\langle\psi, \mathrm{e}^{i \mu x \cdot H^{\prime}} \varphi\right\rangle .
$$

In fact, since the operators $H, H(\nu x)$ and $H_{j}^{\prime}$ are self-adjoint this equality even holds for $\varphi, \psi \in \mathcal{H}$, but we do not need such an extension. This identity, together with the symmetry of $f$, Lemma 4.2.(a), and Proposition 5.2, implies that for $\varphi \in \mathscr{D}_{2}$

$$
\begin{aligned}
& \lim _{r \rightarrow \infty} \frac{1}{2} \int_{0}^{\infty} \mathrm{d} t\left\langle\varphi,\left[\mathrm{e}^{-i t H} f(\Phi / r) \mathrm{e}^{i t H}-\mathrm{e}^{i t H} f(\Phi / r) \mathrm{e}^{-i t H}\right] \varphi\right\rangle \\
& =-\frac{i}{2} \int_{0}^{\infty} \mathrm{d} \mu \int_{\mathbb{R}^{d}} \mathrm{~d} x(\mathscr{F} f)(x)\left\{\left\langle(x \cdot \Phi) \varphi, \mathrm{e}^{i \mu x \cdot H^{\prime}} \varphi\right\rangle-\left\langle\varphi, \mathrm{e}^{-i \mu x \cdot H^{\prime}}(x \cdot \Phi) \varphi\right\rangle\right\} \\
& =-\frac{1}{2} \sum_{j} \int_{0}^{\infty} \mathrm{d} \mu \int_{\mathbb{R}^{d}} \underline{\mathrm{d}} x\left[\mathscr{F}\left(\partial_{j} f\right)\right](x)\left[\left\langle\Phi_{j} \varphi, \mathrm{e}^{i \mu x \cdot H^{\prime}} \varphi\right\rangle+\left\langle\varphi, \mathrm{e}^{i \mu x \cdot H^{\prime}} \Phi_{j} \varphi\right\rangle\right] \\
& =-\frac{1}{2} \sum_{j} \int_{0}^{\infty} \mathrm{d} \mu\left[\left\langle\Phi_{j} \varphi,\left(\partial_{j} f\right)\left(\mu H^{\prime}\right) \varphi\right\rangle+\left\langle\left(\partial_{j} \bar{f}\right)\left(\mu H^{\prime}\right) \varphi, \Phi_{j} \varphi\right\rangle\right] \\
& =t_{f}(\varphi) .
\end{aligned}
$$

(iii) To interchange the limit $\nu \searrow 0$ and the integration over $\mu$ in (5.5), one has to bound $\int_{\mathbb{R}^{d}} \underline{\mathrm{d}} x K(\nu, \mu, x)$ uniformly in $\nu$ by a function in $\mathrm{L}^{1}((0, \infty), \mathrm{d} \mu)$. We begin with the first term of $\int_{\mathbb{R}^{d}} \underline{\mathrm{d}} x K(\nu, \mu, x)$ :

$$
K_{1}(\nu, \mu):=\int_{\mathbb{R}^{d}} \underline{\mathrm{d}} x(\mathscr{F} f)(x)\left\langle\langle\Phi\rangle^{2} \varphi, \frac{1}{\nu}\left(\mathrm{e}^{i \nu x \cdot \Phi}-1\right)\langle\Phi\rangle^{-2} \eta(H(\nu x)) \mathrm{e}^{i \frac{\mu}{\nu}[H(\nu x)-H]} \varphi\right\rangle .
$$

Observe that for each multi-index $\alpha \in \mathbb{N}^{d}$ with $|\alpha| \leq 2$ one has

$$
\left\|\partial_{x}^{\alpha} \frac{1}{\nu}\left(\mathrm{e}^{i \nu x \cdot \Phi}-1\right)\langle\Phi\rangle^{-2}\right\| \leq \text { Const. }\langle x\rangle,
$$

where the derivatives are taken in the strong topology and where the constant is independent of $\nu \in(-1,1)$. Since $\mathscr{F} f \in \mathscr{S}\left(\mathbb{R}^{d}\right)$ it follows that

$$
\left|K_{1}(\nu, \mu)\right| \leq \text { Const., }
$$

and thus $K_{1}(\nu, \mu)$ is bounded uniformly in $\nu$ by a function in $\mathrm{L}^{1}((0,1], \mathrm{d} \mu)$.

For the case $\mu>1$ we first remark that there exists a compact set $J \subset \mathbb{R} \backslash \kappa(H)$ such that $\varphi=E^{H}(J) \varphi$. There also exists $\zeta \in C_{\mathrm{c}}^{\infty}((0, \infty))$ such that $\zeta\left(\left(H^{\prime}\right)^{2}\right) \eta(H)=\eta(H)$ due to Lemma 2.6.(d). It then follows that

$$
\eta(H(\nu x)) \mathrm{e}^{i \frac{\mu}{\nu}[H(\nu x)-H]} \varphi=\zeta\left(H^{\prime}(\nu x)^{2}\right) \eta(H(\nu x)) \mathrm{e}^{i \frac{\mu}{\nu}[H(\nu x)-H]} \varphi .
$$


Moreover, from Assumption 2.3, we also get that

$$
B_{\nu, \mu}^{J}(x) \varphi:=E^{H}(J) \mathrm{e}^{i \frac{\mu}{\nu}[H(\nu x)-H]} E^{H}(J) \varphi=\mathrm{e}^{i \frac{\mu}{\nu}[H(\nu x)-H]} \varphi .
$$

So, $K_{1}(\nu, \mu)$ can be rewritten as

$$
\int_{\mathbb{R}^{d}} \underline{\mathrm{d}} x(\mathscr{F} f)(x)\left\langle\langle\Phi\rangle^{2} \varphi, \frac{1}{\nu}\left(\mathrm{e}^{i \nu x \cdot \Phi}-1\right)\langle\Phi\rangle^{-2} \zeta\left(H^{\prime}(\nu x)^{2}\right) \eta(H(\nu x)) B_{\nu, \mu}^{J}(x) \varphi\right\rangle .
$$

Now, it is easily shown by using Assumption 2.2 and Lemma 2.4 that the function $B_{\nu, \mu}^{J}: \mathbb{R}^{d} \rightarrow \mathscr{B}(\mathcal{H})$ is differentiable with derivative equal to

$$
\left(\partial_{j} B_{\nu, \mu}^{J}\right)(x)=i \mu H_{j}^{\prime}(\nu x) B_{\nu, \mu}^{J}(x) .
$$

Furthermore, the bounded operator

$$
A_{j, \nu}(x):=(\mathscr{F} f)(x) \frac{1}{\nu}\left(\mathrm{e}^{i \nu x \cdot \Phi}-1\right)\langle\Phi\rangle^{-2} H_{j}^{\prime}(\nu x)\left|H^{\prime}(\nu x)\right|^{-2} \zeta\left(H^{\prime}(\nu x)^{2}\right) \eta(H(\nu x))
$$

satisfies for each integer $k \geq 1$ the bound

$$
\left\|A_{j, \nu}(x)\right\| \leq \text { Const. }\langle x\rangle^{-k}
$$

due to Assumption 2.2, Lemma 2.4, Equation (5.6) and the rapid decay of $\mathscr{F} f$. Thus $K_{1}(\nu, \mu)$ can be written as

$$
K_{1}(\nu, \mu)=-i \mu^{-1} \sum_{j} \int_{\mathbb{R}^{d}} \mathrm{~d} x\left\langle\langle\Phi\rangle^{2} \varphi, A_{j, \nu}(x)\left(\partial_{j} B_{\nu, \mu}^{J}\right)(x) \varphi\right\rangle .
$$

Moreover, direct calculations using Equation (5.6) and Proposition 5.1 show that the map $\mathbb{R}^{d} \ni x \mapsto A_{j, \nu}(x) \in$ $\mathscr{B}(\mathcal{H})$ is twice strongly differentiable and satisfies

$$
\left\|\left(\partial_{j} A_{j, \nu}\right)(x)\right\| \leq \text { Const. }\langle x\rangle^{-k}
$$

and

$$
\left\|\partial_{\ell}\left\{\left(\partial_{j} A_{j, \nu}\right) H_{\ell}^{\prime}(\nu \cdot)\left(H^{\prime}(\nu \cdot)\right)^{-2}\right\}(x)\right\| \leq \text { Const. }(1+|\nu|)\langle x\rangle^{-k}
$$

for any integer $k \geq 1$. Therefore one can perform two successive integrations by parts (with vanishing boundary contributions) and obtain

$$
\begin{aligned}
K_{1}(\nu, \mu) & =i \mu^{-1} \sum_{j} \int_{\mathbb{R}^{d}} \underline{\mathrm{d}} x\left\langle\langle\Phi\rangle^{2} \varphi,\left(\partial_{j} A_{j, \nu}\right)(x) B_{\nu, \mu}^{J}(x) \varphi\right\rangle \\
& =-\mu^{-2} \sum_{j, \ell} \int_{\mathbb{R}^{d}} \underline{\mathrm{d}} x\left\langle\langle\Phi\rangle^{2} \varphi, \partial_{\ell}\left\{\left(\partial_{j} A_{j, \nu}\right) H_{\ell}^{\prime}(\nu \cdot)\left(H^{\prime}(\nu \cdot)\right)^{-2}\right\}(x) B_{\nu, \mu}^{J}(x) \varphi\right\rangle .
\end{aligned}
$$

This together with Formula (5.8) implies for each $\nu<1$ and each $\mu>1$ that

$$
\left|K_{1}(\nu, \mu)\right| \leq \text { Const. } \mu^{-2} \text {. }
$$

The combination of the bounds (5.7) and (5.9) shows that $K_{1}(\nu, \mu)$ is bounded uniformly for $\nu<1$ by a function in $\mathrm{L}^{1}((0, \infty), \mathrm{d} \mu)$. Since similar arguments shows that the same holds for the second term of $\int_{\mathbb{R}^{d}} \underline{\mathrm{d}} x K(\nu, \mu, x)$, one can interchange the limit $\nu \searrow 0$ and the integration over $\mu$ in (5.5).

The interchange of the limit $\nu \searrow 0$ and the integration over $x$ in (5.5) is justified by the bound

$$
|K(\nu, \mu, x)| \leq \text { Const. }|x(\mathscr{F} f)(x)|,
$$

which follows from Formula (5.6). 
When the localisation function $f$ is radial, the operator $T_{f}$ is equal to the operator $T$, which is independent of $f$. The next result, which depicts this situation of particular interest, is a direct consequence of Lemma 4.2.(c) and Theorem 5.5

Corollary 5.6. Let $H$ and $\Phi$ satisfy Assumptions 2.2 and 2.3. Let $f \in \mathscr{S}\left(\mathbb{R}^{d}\right)$ be a radial function such that $f=1$ on a neighbourhood of 0 . Then we have for each $\varphi \in \mathscr{D}_{2}$

$$
\lim _{r \rightarrow \infty} \frac{1}{2} \int_{0}^{\infty} \mathrm{d} t\left\langle\varphi,\left[\mathrm{e}^{-i t H} f(\Phi / r) \mathrm{e}^{i t H}-\mathrm{e}^{i t H} f(\Phi / r) \mathrm{e}^{-i t H}\right] \varphi\right\rangle=\langle\varphi, T \varphi\rangle,
$$

with $T$ defined by (5.2).

\section{Interpretation of the integral formula}

This section is devoted to the interpretation of Formula (5.3) and to the description of the sets $\mathscr{D}_{t}$. We begin by stressing some properties of the subspace $\mathcal{K}:=\operatorname{ker}\left(\left(H^{\prime}\right)^{2}\right)$ of $\mathcal{H}$, which plays an important role in the sequel.

Lemma 6.1. (a) The eigenvectors of $H$ belong to $\mathcal{K}$,

(b) If $\varphi \in \mathcal{K}$, then the spectral support of $\varphi$ with respect to $H$ is contained in $\kappa(H)$,

(c) For each $t \geq 0$, the set $\mathcal{K}$ is orthogonal to $\mathscr{D}_{t}$,

(d) For each $t \geq 0$, the set $\mathscr{D}_{t}$ is dense in $\mathcal{H}$ only if $\mathcal{K}$ is trivial.

Proof. As observed in the proof of Lemma 2.6, if $\lambda$ is an eigenvalue of $H$ then one has $E^{H}(\{\lambda\}) H_{j}^{\prime} E^{H}(\{\lambda\})=$ 0 for each $j$. If $\varphi_{\lambda}$ is some corresponding eigenvector, it follows that $H_{j}^{\prime} \varphi_{\lambda}=E^{H}(\{\lambda\}) H_{j}^{\prime} E^{H}(\{\lambda\}) \varphi_{\lambda}=0$. Thus, all eigenvectors of $H$ belong to the kernel of $H_{j}^{\prime}$, and a fortiori to the kernels of $\left(H_{j}^{\prime}\right)^{2}$ and $\left(H^{\prime}\right)^{2}$.

Now, let $\varphi \in \mathcal{K}$ and let $J$ be the minimal closed subset of $\mathbb{R}$ such that $E^{H}(J) \varphi=\varphi$. It follows then from Definition 2.5 that $J \subset \kappa(H)$. This implies that $\varphi \perp \mathscr{D}_{t}$, and thus $\mathcal{K} \perp \mathscr{D}_{t}$. The last statement is a straightforward consequence of point (c).

Let us now proceed to the interpretation of Formula (5.3). We consider first the term $t_{f}(\varphi)$ on the r.h.s., and recall that $f$ is an even element of $\mathscr{S}\left(\mathbb{R}^{d}\right)$ with $f=1$ in a neighbourhood of 0 . We also assume that $f$ is real.

Due to Remark 5.4 with $\varphi \in \mathscr{D}_{1}$, the term $t_{f}(\varphi)$ reduces to the expectation value $\left\langle\varphi, T_{f} \varphi\right\rangle$, with $T_{f}$ given by (5.1). Now, a direct calculation using Formulas (4.1), (4.2), and (5.1) shows that the operators $T_{f}$ and $H$ satisfy in the form sense on $\mathscr{D}_{1}$ the canonical commutation relation

$$
\left[T_{f}, H\right]=i
$$

Therefore, since the group $\left\{\mathrm{e}^{-i t H}\right\}_{t \in \mathbb{R}}$ leaves $\mathscr{D}_{1}$ invariant, the following equalities hold in the form sense on $\mathscr{D}_{1}$ :

$$
T_{f} \mathrm{e}^{-i t H}=\mathrm{e}^{-i t H} T_{f}+\left[T_{f}, \mathrm{e}^{-i t H}\right]=\mathrm{e}^{-i t H} T_{f}-i \int_{0}^{t} \mathrm{~d} s \mathrm{e}^{-i(t-s) H}\left[T_{f}, H\right] \mathrm{e}^{-i s H}=\mathrm{e}^{-i t H}\left(T_{f}+t\right)
$$

In other terms, one has

$$
\left\langle\psi, T_{f} \mathrm{e}^{-i t H} \varphi\right\rangle=\left\langle\psi, \mathrm{e}^{-i t H}\left(T_{f}+t\right) \varphi\right\rangle
$$

for each $\psi, \varphi \in \mathscr{D}_{1}$, and the operator $T_{f}$ satisfies on $\mathscr{D}_{1}$ the so-called infinitesimal Weyl relation in the weak sense $\left[18\right.$, Sec. 3]. Note that we have not supposed that $\mathscr{D}_{1}$ is dense. However, if $\mathscr{D}_{1}$ is dense in $\mathcal{H}$, then the infinitesimal Weyl relation in the strong sense holds:

$$
T_{f} \mathrm{e}^{-i t H} \varphi=\mathrm{e}^{-i t H}\left(T_{f}+t\right) \varphi, \quad \varphi \in \mathscr{D}_{1} .
$$


This relation, also known as $T_{f}$-weak Weyl relation [19, Def. 1.1], has deep implications on the spectral nature of $H$ and on the form of $T_{f}$ in the spectral representation of $H$. Formally, it suggests that $T_{f}=i \frac{\mathrm{d}}{\mathrm{d} H}$, and thus $-i T_{f}$ can be seen as the operator of differentiation with respect to the Hamiltonian $H$. Moreover, being a weak version of the usual Weyl relation, Relation (6.3) also suggests that the spectrum of $H$ may not differ too much from a purely absolutely continuous spectrum. These properties are now discussed more rigorously in particular situations. In the first two cases, the density of $\mathscr{D}_{1}$ in $\mathcal{H}$ is assumed, and so the point spectrum of $H$ is empty by Lemma 6.1.

Case 1 ( $\boldsymbol{T}_{\boldsymbol{f}}$ essentially self-adjoint): If the set $\mathscr{D}_{1}$ is dense in $\mathcal{H}$, and $T_{f}$ is essentially self-adjoint on $\mathscr{D}_{1}$, then it has been shown in [18, Lemma 4] that (6.3) implies that the pair $\left\{\overline{T_{f}}, H\right\}$ satisfies the usual Weyl relation, i.e.

$$
\mathrm{e}^{i s H} \mathrm{e}^{i t \overline{T_{f}}}=\mathrm{e}^{i s t} \mathrm{e}^{i t \overline{T_{f}}} \mathrm{e}^{i s H}, \quad s, t \in \mathbb{R} .
$$

It follows by the Stone-von Neumann theorem [26, VIII.14] that there exists a unitary operator $\mathscr{U}: \mathcal{H} \rightarrow$ $\mathrm{L}^{2}\left(\mathbb{R} ; \mathbb{C}^{N}, \mathrm{~d} \lambda\right)$, with $N$ finite or infinite, such that $\mathscr{U} \mathrm{e}^{i t \overline{T_{f}}} \mathscr{U}^{*}$ is the operator of translation by $t$, and $\mathscr{U} \mathrm{e}^{i s H} \mathscr{U}^{*}$ is the operator of multiplication by $\mathrm{e}^{i s \lambda}$. In terms of the generator $H$, this means that $\mathscr{U} H \mathscr{U}{ }^{*}=\lambda$, where " $\lambda$ " stands for the multiplication operator by $\lambda$ in $\mathrm{L}^{2}\left(\mathbb{R} ; \mathbb{C}^{N}, \mathrm{~d} \lambda\right)$. Therefore the spectrum of $H$ is purely absolutely continuous and covers the whole real line. Moreover, we have for each $\psi \in \mathcal{H}$ and $\varphi \in \mathscr{D}_{1}$

$$
\left\langle\psi, T_{f} \varphi\right\rangle=\left\langle\psi, \overline{T_{f}} \varphi\right\rangle=\int_{\mathbb{R}} \mathrm{d} \lambda\left\langle(\mathscr{U} \psi)(\lambda), i \frac{\mathrm{d}(\mathscr{U} \varphi)}{\mathrm{d} \lambda}(\lambda)\right\rangle_{\mathbb{C}^{N}},
$$

where $\frac{\mathrm{d}}{\mathrm{d} \lambda}$ denotes the distributional derivative (see for instance [2, Rem. 1] for an interpretation of the derivative $\left.\frac{\mathrm{d}}{\mathrm{d} \lambda}\right)$.

Case $2\left(T_{f}\right.$ symmetric): If the set $\mathscr{D}_{1}$ is dense in $\mathcal{H}$, then we know from Proposition 5.2 and Remark 5.4 that $T_{f}$ is symmetric. In such a situation, (6.3) once more implies that the spectrum of $H$ is purely absolutely continuous [19, Thm. 4.4], but it may not cover the whole real line. We expect that the operator $T_{f}$ is still equal to $i \frac{\mathrm{d}}{\mathrm{d} \lambda}$ (on a suitable subspace) in the spectral representation of $H$, but we have not been able to prove it in this generality. However, this property holds in most of the examples presented below. If $T_{f}$ and $H$ satisfy more assumptions, then more can be said (see for instance [33]).

Case 3 ( $\boldsymbol{T}_{f}$ not densely defined): If $\mathscr{D}_{1}$ is not dense in $\mathcal{H}$, then we are not aware of general works using a relation like (6.2) to deduce results on the spectral nature of $H$ or on the form of $T_{f}$ in the spectral representation of $H$. In such a case, we only know from Theorem 3.6 that the spectrum of $H$ is purely absolutely continuous in $\sigma(H) \backslash \kappa(H)$, but we have no general information on the form of $T_{f}$ in the spectral representation of $H$. However, with a suitable additional assumption the analysis can be continued. Indeed, consider the orthogonal decomposition $\mathcal{H}:=\mathcal{K} \oplus \mathcal{G}$, with $\mathcal{K} \equiv \operatorname{ker}\left(\left(H^{\prime}\right)^{2}\right)$. Then the operators $H, H_{j}^{\prime}$, and $H_{k \ell}^{\prime \prime}$ are all reduced by this decomposition, due to the commutation assumption 2.3. If we assume additionally that $T_{f} \mathscr{D}_{1} \subset \mathcal{G}$, then the analysis can be performed in the subspace $\mathcal{G}$.

Since $\mathscr{D}_{1} \subset \mathcal{G}$ by Lemma 6.1, the additional hypothesis allows us to consider the restriction of $T_{f}$ to $\mathcal{G}$, which we denote by $\mathrm{T}_{f}$. Let also $\mathrm{H}, \mathrm{H}_{j}^{\prime}$, and $\mathrm{H}_{k \ell}^{\prime \prime}$ denote the restrictions of the corresponding operators in $\mathcal{G}$. We then set

$$
\mathrm{D}_{t}:=\left\{\varphi \in \mathcal{D}\left(\langle\Phi\rangle^{t}\right) \cap \mathcal{G} \mid \varphi=\eta(\mathrm{H}) \varphi \text { for some } \eta \in C_{\mathrm{c}}^{\infty}(\mathbb{R} \backslash \kappa(H))\right\} \subset \mathcal{G},
$$

and observe that the equality (6.1) holds in the form sense on $D_{1}$. In other words, (6.1) can be considered in the reduced Hilbert space $\mathcal{G}$ instead of $\mathcal{H}$. The interest of the above decomposition comes from the following fact: If $D_{1}$ is dense in $\mathcal{G}$ (which is certainly more likely than in $\mathcal{H}$ ), then $\mathrm{T}_{f}$ is symmetric and the situation reduces to the case 2 with the operators $\mathrm{H}$ and $\mathrm{T}_{f}$. If in addition $\mathrm{T}_{f}$ is essentially self-adjoint on $\mathrm{D}_{1}$, the situation even reduces to the case 1 with the operators $\mathrm{H}_{\text {and }} \mathrm{T}_{f}$. In both situations, the spectrum of $\mathrm{H}$ is purely absolutely continuous. In Section 7, we shall present 2 examples corresponding to these situations.

Remark 6.2. The implicit condition $T_{f} \mathscr{D}_{1} \subset \mathcal{G}$ can be made more explicit. For example, if the collection $\Phi$ is reduced by the decomposition $\mathcal{H}=\mathcal{K} \oplus \mathcal{G}$, then the condition holds (and (5.3) also holds on $\mathrm{D}_{2}$ ). More generally, if $\Phi_{j} \mathscr{D}_{1} \subset \mathcal{G}$ for each $j$, then the condition holds. Indeed, if $\varphi \in \mathscr{D}_{1}$ one knows from Remark 5.4 that $\left(\partial_{j} R_{f}\right)\left(H^{\prime}\right) \varphi \in \mathcal{D}(\langle\Phi\rangle)$, and one can prove similarly that $\left|H^{\prime}\right|^{-1} \varphi \in \mathcal{D}(\langle\Phi\rangle)$. Furthermore, there exists $\eta \in C_{\mathrm{c}}^{\infty}(\mathbb{R} \backslash \kappa(H))$ such that $\left(\partial_{j} R_{f}\right)\left(H^{\prime}\right) \varphi=\eta(H)\left(\partial_{j} R_{f}\right)\left(H^{\prime}\right) \varphi$ and $\left|H^{\prime}\right|^{-1} \varphi=\eta(H)\left|H^{\prime}\right|^{-1} \varphi$, which 
means that both vectors $\partial_{j} R_{f}\left(H^{\prime}\right) \varphi$ and $\left|H^{\prime}\right|^{-1} \varphi$ belong to $\mathscr{D}_{1}$. It follows that $T_{f} \varphi \in \mathcal{G}$ by taking the explicit form (5.1) of $T_{f}$ into account.

Let us now concentrate on the other term in Formula (5.3). If we consider the operators $\Phi_{j}$ as the components of an abstract position operator $\Phi$, then the 1.h.s. of Formula (5.3) has the following meaning: For $r$ fixed, it can be interpreted as the difference of times spent by the evolving state $\mathrm{e}^{-i t H} \varphi$ in the past (first term) and in the future (second term) within the region defined by the localisation operator $f(\Phi / r)$. Thus, Formula (5.3) shows that this difference of times tends as $r \rightarrow \infty$ to the expectation value in $\varphi$ of the operator $T_{f}$.

On the other hand, let us consider a quantum scattering pair $\{H, H+V\}$, with $V$ an appropriate perturbation of $H$. Let us also assume that the corresponding scattering operator $S$ is unitary, and recall that $S$ commute with $H$. In this framework, the global time delay $\tau(\varphi)$ for the state $\varphi$ defined in terms of the localisation operators $f(\Phi / r)$ can usually be reexpressed as follows: it is equal to the 1.h.s. of (5.3) minus the same quantity with $\varphi$ replaced by $S \varphi$. Therefore, if $\varphi$ and $S \varphi$ are elements of $\mathscr{D}_{2}$, then the time delay for the scattering pair $\{H, H+V\}$ should satisfy the equation

$$
\tau(\varphi)=-\left\langle\varphi, S^{*}\left[T_{f}, S\right] \varphi\right\rangle .
$$

In addition, if $T_{f}$ acts in the spectral representation of $H$ as a differential operator $i \frac{\mathrm{d}}{\mathrm{d} H}$, then $\tau(\varphi)$ would verify, in our complete abstract setting, the Eisenbud-Wigner formula

$$
\tau(\varphi)=\left\langle\varphi,-i S^{*} \frac{\mathrm{d} S}{\mathrm{~d} H} \varphi\right\rangle .
$$

Summing up, as soon as the position operator $\Phi$ and the operator $H$ satisfy Assumptions 2.2 and 2.3, then our study establishes a preliminary relation between time operators $T_{f}$ given by (5.1) and the theory of quantum time delay. Many concrete examples discussed in the literature $[2,3,4,15,20,35,37]$ turn out to fit in the present framework, and several old or new examples are presented in the following section. Further investigations in relation with the abstract Formula (6.4) will be considered elsewhere.

Now, most of the above discussion depends on the size of $\mathscr{D}_{1}$ in $\mathcal{H}$, and implicitly on the size of $\kappa(H)$ in $\sigma(H)$. We collect some information about these sets. It has been proved in Lemma 2.6.(d) that $\kappa(H)$ is closed and corresponds to the complement in $\sigma(H)$ of the Mourre set (see the comment after Definition 3.4). It always contains the eigenvalues of $H$. Furthermore, since the spectrum of $H$ is absolutely continuous on $\sigma(H) \backslash \kappa(H)$, the support of the singularly continuous spectrum, if any, is contained in $\kappa(H)$. In particular, if $\kappa(H)$ is discrete, then $H$ has no singularly continuous spectrum. Thus, the determination of the size of $\kappa(H)$ is an important issue for the spectral analysis of $H$. More will be said in the concrete examples of the next section.

Let us now turn to the density properties of the sets $\mathscr{D}_{t}$. For this, we recall that a subset $K \subset \mathbb{R}$ is said to be uniformly discrete if

$$
\inf \{|x-y| \mid x, y \in K \text { and } x \neq y\}>0 .
$$

Lemma 6.3. Assume that $\kappa(H)$ is uniformly discrete. Then

(a) $\mathscr{D}_{0}$ is dense in $\mathcal{H}_{\mathrm{ac}}(H)$,

(b) If $\sigma_{\mathrm{p}}(H)=\varnothing$ and if $H$ is of class $C^{k}(\Phi)$ for some integer $k$, then $\mathscr{D}_{t}$ is dense in $\mathcal{H}$ for any $t \in[0, k)$.

Proof. (a) Let $\varphi \in \mathcal{H}_{\mathrm{ac}}(H)$ and $\varepsilon>0$. Then there exists a finite interval $[a, b]$ such that $\left\|\left[1-E^{H}([a, b])\right] \varphi\right\| \leq$ $\varepsilon / 2$. Since $\kappa(H)$ is uniformly discrete, the set $\kappa(H) \cap(a, b)$ contains only a finite number $N$ of points $x_{1}<x_{2}<$ $\cdots<x_{N}$. Let us set $x_{0}:=a$ and $x_{N+1}:=b$. Since $\varphi \in \mathcal{H}_{\mathrm{ac}}$, there exists $\delta>0$ such that $x_{j}+\delta<x_{j+1}-\delta$ for each $j \in\{0, \ldots, N\}$, and $\left\|E^{H}\left(L_{\delta}\right) \varphi\right\| \leq \varepsilon / 2$, where

$$
L_{\delta}:=\left\{x \in[a, b]|| x-x_{j} \mid \leq \delta \text { for each } j=0,1, \ldots, N+1\right\} .
$$

Now, for any $j \in\{0, \ldots, N\}$ there exist $\eta_{j}, \widetilde{\eta}_{j} \in C_{\mathrm{c}}^{\infty}\left(\left(x_{j}, x_{j+1}\right) ;[0,1]\right)$ such that $\widetilde{\eta}_{j}(x)=1$ for $x \in\left[x_{j}+\right.$ $\left.\delta, x_{j+1}-\delta\right]$ and $\eta_{j} \widetilde{\eta}_{j}=\widetilde{\eta}_{j}$. Therefore, if $\eta:=\sum_{j=0}^{N} \eta_{j}, \widetilde{\eta}:=\sum_{j=0}^{N} \widetilde{\eta}_{j}$ and $\psi:=\widetilde{\eta}(H) \varphi$, one verifies that 
$\eta \in C_{\mathrm{c}}^{\infty}((a, b) ;[0,1]) \subset C_{\mathrm{c}}^{\infty}(\mathbb{R} \backslash \kappa(H))$ and that $\psi=\eta(H) \psi$, which imply that $\psi \in \mathscr{D}_{0}$. Moreover, one has

$$
\begin{aligned}
\|\varphi-\psi\| & \leq\left\|[1-\widetilde{\eta}(H)] E^{H}([a, b]) \varphi\right\|+\left\|[1-\widetilde{\eta}(H)]\left[1-E^{H}([a, b])\right] \varphi\right\| \\
& \leq\left\|[1-\widetilde{\eta}(H)] E^{H}\left(L_{\delta}\right) \varphi\right\|+\left\|\left[1-E^{H}([a, b])\right] \varphi\right\| \\
& \leq \frac{\varepsilon}{2}+\frac{\varepsilon}{2} .
\end{aligned}
$$

Thus $\|\varphi-\psi\| \leq \varepsilon$ for $\psi \in \mathscr{D}_{0}$, and the claim is proved.

(b) If $\sigma_{\mathrm{p}}(H)=\varnothing$, then it follows from the above discussion that $\mathcal{H}_{\mathrm{ac}}(H)=\mathcal{H}$. In view of what precedes, it is enough to show that the vector $\psi \equiv \widetilde{\eta}(H) \varphi$ of point (a) belongs to $\mathcal{D}\left(\langle\Phi\rangle^{t}\right.$ ): The operator $\widetilde{\eta}(H)$ belongs to $C^{k}(\Phi)$, since $H$ is of class $C^{k}(\Phi)$ and $\widetilde{\eta} \in C_{\mathrm{c}}^{\infty}(\mathbb{R})$ (see [1, Thm. 6.2.5]). So, we obtain from [1, Prop. 5.3.1] that $\langle\Phi\rangle^{t} \widetilde{\eta}(H)\langle\Phi\rangle^{-t}$ is bounded on $\mathcal{H}$, which implies the claim.

\section{Examples}

In this section we show that Assumptions 2.2 and 2.3 are satisfied in various general situations. In these situations all the results of the preceding sections such as Theorem 3.6 or Formula (5.3) hold. However, it is usually impossible to determine explicitly the set $\kappa(H)$ when the framework is too general. Therefore, we also illustrate our approach with some concrete examples for which everything can be computed explicitly. When possible, we also relate these examples with the different cases presented in Section 6. For that purpose, we shall always assume that $f$ is a real and even function in $\mathscr{S}\left(\mathbb{R}^{d}\right)$ with $f=1$ on a neighbourhood of 0 .

The configuration space of the system under consideration will sometimes be $\mathbb{R}^{n}$, and the corresponding Hilbert space $\mathrm{L}^{2}\left(\mathbb{R}^{n}\right)$. In that case, the notations $Q \equiv\left(Q_{1}, \ldots, Q_{n}\right)$ and $P \equiv\left(P_{1}, \ldots, P_{n}\right)$ refer to the families of position operators and momentum operators. More precisely, for suitable $\varphi \in \mathrm{L}^{2}\left(\mathbb{R}^{n}\right)$ and each $j \in\{1, \ldots, n\}$, we have $\left(Q_{j} \varphi\right)(x)=x_{j} \varphi(x)$ and $\left(P_{j} \varphi\right)(x)=-i\left(\partial_{j} \varphi\right)(x)$ for each $x \in \mathbb{R}^{n}$.

\section{1 $H^{\prime}$ constant}

Suppose that $H$ is of class $C^{1}(\Phi)$, and assume that there exists $v \in \mathbb{R}^{d} \backslash\{0\}$ such that $H^{\prime}=v$. Then $H$ is of class $C^{\infty}(\Phi)$, Assumption 2.2 is directly verified, and one has on $\mathcal{D}(H)$

$$
H(x)=H(0)+\int_{0}^{1} \mathrm{~d} t\left(x \cdot H^{\prime}(t x)\right)=H+\int_{0}^{1} \mathrm{~d} t \mathrm{e}^{-i t x \cdot \Phi}\left(x \cdot H^{\prime}\right) \mathrm{e}^{-i t x \cdot \Phi}=H+x \cdot v .
$$

This implies Assumption 2.3. Furthemore $\kappa(H)=\varnothing$, and $\sigma(H)=\sigma_{\text {ac }}(H)$ due to Theorem 3.6. So, the set $\mathscr{D}_{t}$ is dense in $\mathcal{H}$ for each $t \geq 0$, due to Lemma 6.3.(b). The operator $R_{f}^{\prime}\left(H^{\prime}\right)$ reduces to the constant vector $R_{f}^{\prime}(v)$. Therefore, we have the equality $T_{f}=-R_{f}^{\prime}(v) \cdot \Phi$ on $\mathscr{D}_{1}$, and it is easily shown that $T_{f}$ is essentially self-adjoint on $\mathscr{D}_{1}$. It follows from the case 1 of Section 6 that the spectrum of $H$ covers the whole real line, and there exists a unitary operator $\mathscr{U}: \mathcal{H} \rightarrow \mathrm{L}^{2}\left(\mathbb{R} ; \mathbb{C}^{N}, \mathrm{~d} \lambda\right)$ such that

$$
\left\langle\psi, T_{f} \varphi\right\rangle=\int_{\mathbb{R}} \mathrm{d} \lambda\left\langle(\mathscr{U} \psi)(\lambda), i \frac{\mathrm{d}(\mathscr{U} \varphi)}{\mathrm{d} \lambda}(\lambda)\right\rangle_{\mathbb{C}^{N}}
$$

for each $\psi \in \mathcal{H}$ and $\varphi \in \mathscr{D}_{1}$.

Typical examples of operators $H$ and $\Phi$ fitting into this construction are Friedrichs-type Hamiltonians and position operators. For illustration, we mention the case $H:=v \cdot P+V(Q)$ and $\Phi:=Q$ in $\mathrm{L}^{2}\left(\mathbb{R}^{d}\right)$, with $v \in \mathbb{R}^{d} \backslash\{0\}$ and $V \in \mathrm{L}^{\infty}\left(\mathbb{R}^{d} ; \mathbb{R}\right.$ ) (see also [37, Sec. 5] for informations on quantum time delay in a similar case).

Stark Hamiltonians and momentum operators also fit into the construction, i.e. $H:=P^{2}+v \cdot Q$ in $\mathrm{L}^{2}\left(\mathbb{R}^{d}\right)$ with $v \in \mathbb{R}^{d} \backslash\{0\}$, and $\Phi:=P$. We refer to [25, 29, 30] for previous accounts on the theory of time operators and quantum time delay in similar situations.

Note that these first two examples are interesting since the operators $H$ contain not only a kinetic part, but also a potential perturbation. 
Another example is provided by the Jacobi operator related to the family of Hermite polynomials (see [32, Appendix A] for details). In the Hilbert space $\mathcal{H}:=\ell^{2}(\mathbb{N})$, consider the Jacobi operator given for $\varphi \in \mathcal{H}$ by

$$
(H \varphi)(n):=\frac{\sqrt{n-1}}{2} \varphi(n-1)+\frac{\sqrt{n}}{2} \varphi(n+1)
$$

with the convention that $\varphi(0)=0$. The operator $H$ is essentially self-adjoint on $\ell_{0}^{2}$, the subspace of sequences in $\mathcal{H}$ with only finitely many non-zero components. As operator $\Phi$ (with one component), take

$$
(\Phi \varphi)(n):=-i\{\sqrt{n-1} \varphi(n-1)-\sqrt{n} \varphi(n+1)\},
$$

which is also essentially self-adjoint on $\ell_{0}^{2}$. Then $H$ is of class $C^{1}(\Phi)$ and $H^{\prime} \equiv i[H, \Phi]=1$, and so the preceding results hold.

\section{2 $H^{\prime}=H$}

Suppose that $\Phi$ has only one component, and assume that $H$ is $\Phi$-homogeneous of degree 1, i.e. $H(x) \equiv$ $\mathrm{e}^{-i x \Phi} H \mathrm{e}^{i x \Phi}=\mathrm{e}^{x} H$ for all $x \in \mathbb{R}$. This implies that $H$ is of class $C^{\infty}(\Phi)$ and that $H^{\prime}=H$. So, Assumptions 2.2 and 2.3 are readily verified. Moreover, since $\kappa(H)=\{0\}$, Theorem 3.6 implies that $H$ is purely absolutely continuous except at the origin, where it may have the eigenvalue 0 .

Now, let us show that the formal formula of Remark 5.3 holds in this case. For any $\varphi \in \mathscr{D}_{1}$ one has by Remark 5.4 that $R_{f}^{\prime}\left(H^{\prime}\right) \varphi \equiv R_{f}^{\prime}(H) \varphi$ belongs to $\mathcal{D}(\Phi)$. On another hand, we have

$$
\Phi \varphi=\{H \Phi+[\Phi, H]\} H^{-1} \varphi=H(\Phi+i) H^{-1} \varphi,
$$

which implies that $R_{f}^{\prime}(H) \Phi \varphi=R_{f}^{\prime}\left(\frac{H}{|H|}\right) \frac{H}{|H|}(\Phi+i) H^{-1} \varphi \in \mathcal{H}$. In consequence, the operator

$$
T_{f}=-\frac{1}{2}\left(\Phi R_{f}^{\prime}(H)+R_{f}^{\prime}(H) \Phi\right)
$$

is well-defined on $\mathscr{D}_{1}$. In particular, if 0 is not an eigenvalue of $H$, then $T_{f}$ is a symmetric operator and the discussion of the case 2 of Section 6 is relevant (if $T_{f}$ is essentially self-adjoint, the case 1 is relevant).

We now give two examples of pairs $\{H, \Phi\}$ satisfying the preceding assumptions. Other examples are presented in [8, Sec. 10]. Suppose that $H:=P^{2}$ is the free Schrödinger operator in $\mathcal{H}:=\mathrm{L}^{2}\left(\mathbb{R}^{n}\right)$ and $\Phi:=$ $\frac{1}{4}(Q \cdot P+P \cdot Q)$ is the generator of dilations in $\mathcal{H}$. Then the relation $\mathrm{e}^{-i x \Phi} H \mathrm{e}^{i x \Phi}=\mathrm{e}^{x} H$ is satisfied, $\sigma(H)=\sigma_{\mathrm{ac}}(H)=[0, \infty)$. Furthermore, for $\psi \in \mathcal{H}$ and $\varphi \in \mathscr{F} C_{\mathrm{c}}^{\infty}\left(\mathbb{R}^{n} \backslash\{0\}\right) \subset \mathscr{D}_{1}$ a direct calculation using Formula (4.1) shows that

$$
\left\langle\psi, T_{f} \varphi\right\rangle=\left\langle\psi, \frac{1}{4}\left(Q \cdot P P^{-2}+P P^{-2} \cdot Q\right) \varphi\right\rangle=\int_{0}^{\infty} \mathrm{d} \lambda\left\langle(\mathscr{U} \psi)(\lambda), i \frac{\mathrm{d}(\mathscr{U} \varphi)}{\mathrm{d} \lambda}(\lambda)\right\rangle_{\mathrm{L}^{2}\left(\mathbb{S}^{n-1}\right)},
$$

where $\mathscr{U}: \mathcal{H} \rightarrow \int_{[0, \infty)}^{\oplus} \mathrm{d} \lambda \mathrm{L}^{2}\left(\mathbb{S}^{n-1}\right)$ is the spectral transformation for $P^{2}$. This example corresponds to the case 2 of Section 6.

Another example of $\Phi$-homogeneous operator is provided by the Jacobi operator related to the family of Laguerre polynomials (see [32, Appendix A] for details). In the Hilbert space $\mathcal{H}:=\ell^{2}(\mathbb{N})$, consider the Jacobi operator given for $\varphi \in \mathcal{H}$ by

$$
(H \varphi)(n):=(n-1) \varphi(n-1)+(2 n-1) \varphi(n)+n \varphi(n+1),
$$

with the convention that $\varphi(0)=0$. The operator $H$ is essentially self-adjoint on $\ell_{0}^{2}$. As operator $\Phi$ (with one component), take

$$
(\Phi \varphi)(n):=-\frac{i}{2}\{(n-1) \varphi(n-1)-n \varphi(n+1)\} .
$$

Then one has $H^{\prime} \equiv i[H, \Phi]=H$, which implies that $H$ is $\Phi$-homogeneous of degree 1 and so the preceding results hold. 


\subsection{Dirac operator}

In the Hilbert space $\mathcal{H}:=\mathrm{L}^{2}\left(\mathbb{R}^{3} ; \mathbb{C}^{4}\right)$ we consider the Dirac operator for a spin- $\frac{1}{2}$ particle of mass $m>0$

$$
H:=\alpha \cdot P+\beta m,
$$

where $\alpha \equiv\left(\alpha_{1}, \alpha_{2}, \alpha_{3}\right)$ and $\beta$ denote the usual $4 \times 4$ Dirac matrices. It is known that $H$ has domain $\mathcal{H}^{1}\left(\mathbb{R}^{3} ; \mathbb{C}^{4}\right)$, that $|H|=\left(P^{2}+m^{2}\right)^{1 / 2}$ and that $\sigma(H)=\sigma_{\text {ac }}(H)=(-\infty,-m] \cup[m, \infty)$.

We also let $\Phi:=\mathscr{U}_{\mathrm{FW}}^{-1} Q \mathscr{U}_{\mathrm{FW}} \equiv Q_{\mathrm{NW}}$ be the Wigner-Newton position operator, with $\mathscr{U}_{\mathrm{FW}}$ the usual Foldy-Wouthuysen transformation [34, Sec. 1.4.3]. Then a direct calculation shows that

$$
H(x)=\sqrt{\frac{(P+x)^{2}+m^{2}}{P^{2}+m^{2}}} H
$$

for each $x \in \mathbb{R}^{3}$, and thus Assumptions 2.2 and 2.3 are clearly satisfied. Furthermore, since $H_{j}^{\prime}=P_{j} H^{-1}$ for each $j=1,2,3$, it follows that

$$
\left(H^{\prime}\right)^{2}=P^{2} H^{-2}=\left(H^{2}-m^{2}\right) H^{-2} .
$$

Clearly, ker $\left(\left(H^{\prime}\right)^{2}\right)=\{0\}$ and one infers from Definition 2.5 that $\kappa(H)=\{ \pm m\}$, and from Lemma 6.3.(b) that the sets

$$
\mathscr{D}_{t}=\left\{\varphi \in \mathscr{U}_{\mathrm{FW}}^{-1} \mathcal{D}\left(\langle Q\rangle^{t}\right) \mid \eta(H) \varphi=\varphi \text { for some } \eta \in C_{\mathrm{c}}^{\infty}(\mathbb{R} \backslash\{ \pm m\})\right\},
$$

are dense in $\mathcal{H}$. So the discussion of the case 2 of Section 6 is relevant.

We now show that the formal formula of Remark 5.3 holds if $f$ is radial. Indeed, each $\varphi \in \mathscr{D}_{1}$ satisfies $\varphi=\eta(H) \mathscr{U}_{\mathrm{FW}}^{-1} \psi$ for some $\eta \in C_{\mathrm{c}}^{\infty}(\mathbb{R} \backslash\{ \pm m\})$ and some $\psi \in \mathcal{D}(\langle Q\rangle)$. So, we have

$$
H^{\prime}\left(H^{\prime}\right)^{-2} \cdot Q_{\mathrm{NW}} \varphi=P P^{-2} H \cdot \mathscr{U}_{\mathrm{FW}}^{-1} Q \mathscr{U}_{\mathrm{FW}} \eta(H) \mathscr{U}_{\mathrm{FW}}^{-1} \psi=\mathscr{U}_{\mathrm{FW}}^{-1} P P^{-2} \beta|H| \cdot Q \eta(\beta|H|) \psi \in \mathcal{H},
$$

and the operator $T$ of (5.2) is symmetric and can be written on $\mathscr{D}_{1}$ in the simpler form

$$
T=\frac{1}{2}\left\{Q_{\mathrm{NW}} \cdot H^{\prime}\left(H^{\prime}\right)^{-2}+H^{\prime}\left(H^{\prime}\right)^{-2} \cdot Q_{\mathrm{NW}}\right\} \equiv \frac{1}{2}\left\{Q_{\mathrm{NW}} \cdot P P^{-2} H+P P^{-2} H \cdot Q_{\mathrm{NW}}\right\} .
$$

Now let $h: \mathbb{R}^{3} \rightarrow \mathbb{R}$ be defined by $h(\xi):=\left(\xi^{2}+m^{2}\right)^{1 / 2}$. Then it is known that $\mathscr{U}_{\mathrm{FW}} H \mathscr{U}_{\mathrm{FW}}^{-1}=\beta h(P)$, and a direct calculation shows that

$$
\mathscr{U}_{\mathrm{FW}} T \mathscr{U}_{\mathrm{FW}}^{-1}=\frac{1}{2} \beta\left\{Q \cdot P P^{-2}\left(P^{2}+m^{2}\right)^{1 / 2}+P P^{-2}\left(P^{2}+m^{2}\right)^{1 / 2} \cdot Q\right\}=\frac{1}{2} \beta\left\{Q \cdot \frac{h^{\prime}(P)}{h^{\prime}(P)^{2}}+\frac{h^{\prime}(P)}{h^{\prime}(P)^{2}} \cdot Q\right\}
$$

on $\mathscr{U}_{\mathrm{FW}} \mathscr{D}_{1}$. Furthermore there exists a spectral transformation $\mathscr{U}_{0}: \mathrm{L}^{2}\left(\mathbb{R}^{3}\right) \rightarrow \int_{[m, \infty)}^{\oplus} \mathrm{d} \lambda \mathrm{L}^{2}\left(\mathbb{S}^{2}\right)$ for $h(P)$ such that

$$
\mathscr{U}_{0}\left\{Q \cdot \frac{h^{\prime}(P)}{h^{\prime}(P)^{2}}+\frac{h^{\prime}(P)}{h^{\prime}(P)^{2}} \cdot Q\right\} \mathscr{U}_{0}^{-1}
$$

is equal to the operator $2 i \frac{\mathrm{d}}{\mathrm{d} \lambda}$ of differentiation with respect to the spectral parameter $\lambda$ of $h(P)$ (see [37, Lemma 3.6] for a precise statement). Combining the preceding transformations we obtain for each $\psi \in \mathcal{H}$ and $\varphi \in \mathscr{D} 1$ that

$$
\langle\psi, T \varphi\rangle=\int_{\sigma(H)} \mathrm{d} \lambda\left\langle(\mathscr{U} \psi)(\lambda), i \frac{\mathrm{d}(\mathscr{U} \varphi)}{\mathrm{d} \lambda}(\lambda)\right\rangle_{\mathrm{L}^{2}\left(\mathbb{S}^{2} ; \mathbb{C}^{2}\right)},
$$

where $\mathscr{U}: \mathcal{H} \rightarrow \int_{\sigma(H)}^{\oplus} \mathrm{d} \lambda \mathrm{L}^{2}\left(\mathbb{S}^{2} ; \mathbb{C}^{2}\right)$ is the spectral transformation for the free Dirac operator $H$.

\subsection{Convolution operators on locally compact groups}

This example is partially inspired from [22], where the spectral nature of convolution operators on locally compact groups is studied.

Let $G$ be a locally compact group with identity $e$ and a left Haar measure $\rho$. In the Hilbert space $\mathcal{H}:=$ $\mathrm{L}^{2}(G, \mathrm{~d} \rho)$ we consider the operator $H_{\mu}$ of convolution by $\mu \in \mathrm{M}(G)$, where $\mathrm{M}(G)$ is the set of complex bounded Radon measures on $G$. Namely, for $\varphi \in \mathcal{H}$ one sets

$$
\left(H_{\mu} \varphi\right)(g):=(\mu * \varphi)(g) \equiv \int_{G} \mathrm{~d} \mu(h) \varphi\left(h^{-1} g\right) \quad \text { for a.e. } g \in G,
$$


where the notation a.e. stands for "almost everywhere" and refers to the Haar measure $\rho$. The operator $H_{\mu}$ is bounded with norm $\left\|H_{\mu}\right\| \leq|\mu|(G)$, and it is self-adjoint if $\mu$ is symmetric, i.e. $\mu(E)=\overline{\mu\left(E^{-1}\right)}$ for each Borel subset $E$ of $G$. For simplicity, we also assume that $\mu$ is central and with compact support, where central means that $\mu\left(h^{-1} E h\right)=\mu(E)$ for each $h \in G$ and each Borel subset $E$ of $G$.

We recall that given two measures $\mu, \nu \in \mathrm{M}(G)$, their convolution $\mu * \nu \in \mathrm{M}(G)$ is defined by the relation [11, Eq. 2.34]

$$
\int_{G} \mathrm{~d}(\mu * \nu)(g) \psi(g):=\int_{G} \int_{G} \mathrm{~d} \mu(g) \mathrm{d} \nu(h) \psi(g h) \quad \forall \psi \in C_{0}(G),
$$

where $C_{0}(G)$ denotes the $C^{*}$-algebra of continuous complex functions on $G$ vanishing at infinity. If $\mu \in \operatorname{M}(G)$ has compact support and $\zeta: G \rightarrow \mathbb{C}$ is continuous, then the linear functional

$$
C_{0}(G) \ni \psi \mapsto \int_{G} \mathrm{~d} \mu(g) \zeta(g) \psi(g) \in \mathbb{C}
$$

is bounded, and there exists a unique measure with compact support associated with it, due to the Riesz-Markov representation theorem. We write $\zeta \mu$ for this measure.

A natural choice for the family of operators $\Phi \equiv\left(\Phi_{1}, \ldots, \Phi_{d}\right)$ are, if they exist, real characters $\Phi_{j} \in$ $\operatorname{Hom}(G ; \mathbb{R})$, i.e. continuous group morphisms from $G$ to $\mathbb{R}$. With this choice, one obtains that

$$
\left[H_{\mu}(x) \varphi\right](g) \equiv\left(\mathrm{e}^{-i x \cdot \Phi} H_{\mu} \mathrm{e}^{i x \cdot \Phi} \varphi\right)(g)=\int_{G} \mathrm{~d} \mu(h) \mathrm{e}^{-i x \cdot \Phi(h)} \varphi\left(h^{-1} g\right)
$$

for each $x \in \mathbb{R}^{d}, \varphi \in \mathcal{H}$, and a.e. $g \in G$. Namely, $H_{\mu}(x)$ is equal to the operator of convolution by the measure $\mathrm{e}^{-i x \cdot \Phi} \mu$, i.e. $H_{\mu}(x)=H_{\mathrm{e}^{-i x} \cdot \Phi}$. Since $\mu$ has compact support and each $\Phi_{j}$ is continuous, this implies that $H_{\mu}$ is of class $C^{\infty}(\Phi)$. So Assumption 2.2 is satisfied. Furthermore, the commutativity of central measures with respect to the convolution product implies that $\mu * \mathrm{e}^{-i x \cdot \Phi} \mu=\mathrm{e}^{-i x \cdot \Phi} \mu * \mu$ or equivalently that $H H(x)=$ $H(x) H$. So Assumption 2.3 is satisfied. Finally, the equality $H_{\mu}(x)=H_{\mathrm{e}^{-i x} \cdot \Phi}{ }_{\mu}$ readily implies that $\left(H_{\mu}^{\prime}\right)_{j}=$ $H_{-i \Phi_{j} \mu}$.

Since both Assumptions 2.2 and 2.3 are satisfied, the general results of the previous sections apply. However, it is very complicated to describe the set $\kappa\left(H_{\mu}\right)$ in the present generality. Therefore, we shall now assume that the group $G$ is abelian in order to use the Fourier transformation to determine some properties of $\kappa\left(H_{\mu}\right)$. So let us assume that $G$ is a locally compact abelian group. Then any measure on $G$ is automatically central, and thus we only need to suppose that $\mu$ is symmetric and with compact support. For a suitably normalised Haar measure $\rho_{\wedge}$ on the dual group $\widehat{G}$, the Fourier transformation $\mathscr{F}$ defines a unitary isomorphism from $\mathcal{H}$ onto $\mathrm{L}^{2}\left(\widehat{G}, \mathrm{~d} \rho_{\wedge}\right)$. It maps unitarily $H_{\mu}$ on the operator $M_{m}$ of multiplication with the bounded continuous real function $m:=\mathscr{F}(\mu)$ on $\widehat{G}$. Furthermore, one has

$$
\sigma\left(H_{\mu}\right)=\sigma\left(M_{m}\right)=\overline{m(\widehat{G})}, \quad \sigma_{\mathrm{p}}\left(H_{\mu}\right)=\sigma_{\mathrm{p}}\left(M_{m}\right)=\overline{\left\{s \in \mathbb{R} \mid \rho_{\wedge}\left(m^{-1}(s)\right)>0\right\}}
$$

where the overlines denote the closure in $\mathbb{R}$.

Let us recall that there is an almost canonical identification of $\operatorname{Hom}(G, \mathbb{R})$ with the vector space $\operatorname{Hom}(\mathbb{R}, \widehat{G})$ of all continuous one-parameter subgroups of $\widehat{G}$. Given the real character $\Phi_{j}$, we denote by $\Upsilon_{j} \in \operatorname{Hom}(\mathbb{R}, \widehat{G})$ the unique element satisfying

$$
\left\langle g, \Upsilon_{j}(t)\right\rangle=\mathrm{e}^{i t \Phi_{j}(g)} \quad \text { for all } t \in \mathbb{R} \text { and } g \in G,
$$

where $\langle\cdot, \cdot\rangle: G \times \widehat{G} \rightarrow \mathbb{C}$ is the duality between $G$ and $\widehat{G}$.

Definition 7.1. A function $m: \widehat{G} \rightarrow \mathbb{C}$ is differentiable at $\xi \in \widehat{G}$ along the one-parameter subgroup $\Upsilon_{j} \in$ $\operatorname{Hom}(\mathbb{R}, \widehat{G})$ if the function $\mathbb{R} \ni t \mapsto m\left(\xi+\Upsilon_{j}(t)\right) \in \mathbb{C}$ is differentiable at $t=0$. In such a case we write $\left(d_{j} m\right)(\xi)$ for $\left.\frac{\mathrm{d}}{\mathrm{d} t} m\left(\xi+\Upsilon_{j}(t)\right)\right|_{t=0}$. Higher order derivatives, when existing, are denoted by $d_{j}^{k} m, k \in \mathbb{N}$. 
We refer to [28] for more details on differential calculus on locally compact groups. Here we only note that (since $\mu$ has compact support) the function $m=\mathscr{F}(\mu)$ is differentiable at any point $\xi$ along the one-parameter subgroup $\Upsilon_{j}$, and $-i \mathscr{F}\left(\Phi_{j} \mu\right)=d_{j} m$ [28, p. 68]. This implies that the operator $\left(H_{\mu}^{\prime}\right)_{j}$ is mapped unitarily by $\mathscr{F}$ on the multiplication operator $M_{d_{j} m}$, and thus $\left(H_{\mu}^{\prime}\right)^{2}$ is unitarily equivalent to the operator of multiplication by the function $\sum_{j}\left(d_{j} m\right)^{2}$. It follows that

$$
\kappa\left(H_{\mu}\right) \supset\left\{\lambda \in \mathbb{R} \mid \exists \xi \in \widehat{G} \text { such that } m(\xi)=\lambda \text { and } \sum_{j}\left(d_{j} m\right)(\xi)^{2}=0\right\} .
$$

This property of $\kappa\left(H_{\mu}\right)$ suggests a way to justify the formal formula of Remark 5.3 and to write nice formulas for the operator $T$ given by (5.2). Indeed, since $\mathscr{F} \Phi_{j} \mathscr{F}^{-1}$ acts as the differential operator $i d_{j}$ in $\mathrm{L}^{2}\left(\widehat{G}, \mathrm{~d} \rho_{\wedge}\right)$, it follows that $\Phi_{j}$ leaves invariant the complement of the support of the functions on which it acts. Therefore, the set $\Phi_{j} \mathscr{D}_{1} \equiv \mathscr{F}^{-1}\left(i d_{j}\right) \mathscr{F}_{\mathscr{D}_{1}}$ is included in the domain of the operator

$$
\frac{\left(H_{\mu}^{\prime}\right)_{j}}{\left(H_{\mu}^{\prime}\right)^{2}} \equiv \mathscr{F}^{-1} \frac{M_{d_{j} m}}{M_{\sum_{k}\left(d_{k} m\right)^{2}}} \mathscr{F} .
$$

Thus the formula (5.2) takes the form

$$
T=\frac{1}{2} \sum_{j}\left\{\Phi_{j} \frac{H_{-i \Phi_{j} \mu}}{\sum_{k}\left(H_{-i \Phi_{k} \mu}\right)^{2}}+\frac{H_{-i \Phi_{j} \mu}}{\sum_{k}\left(H_{-i \Phi_{k} \mu}\right)^{2}} \Phi_{j}\right\}
$$

on $\mathscr{D}_{1}$, or alternatively the form

$$
\mathscr{F} T \mathscr{F}^{-1}=\frac{i}{2} \sum_{j}\left\{d_{j} \frac{M_{d_{j} m}}{M_{\sum_{k}\left(d_{k} m\right)^{2}}}+\frac{M_{d_{j} m}}{M_{\sum_{k}\left(d_{k} m\right)^{2}}} d_{j}\right\}
$$

on $\mathscr{F} \mathscr{D}_{1}$ (note that the last expression is well-defined on $\mathscr{F} \mathscr{D}_{1}$, since $m=\mathscr{F}(\mu)$ is of class $C^{2}$ in the sense of Definition 7.1).

In simple situations, everything can be calculated explicitly. For instance, when $G=\mathbb{Z}^{d}$, the Haar measure $\rho$ is the counting measure, and the most natural real characters $\Phi_{j}$ are the position operators given by

$$
\left(\Phi_{j} \varphi\right)(g):=g_{j} \varphi(g), \quad \varphi \in \mathrm{L}^{2}\left(\mathbb{Z}^{d}\right),
$$

where $g_{j}$ is the $j$-th component of $g \in \mathbb{Z}^{d}$. The operators $H_{\mu}$ and $\left(H_{\mu}^{\prime}\right)^{2}$ are unitarily equivalent to multiplication operators on $\widehat{G}=(-\pi, \pi]^{d}$. Since the measures $\mu$ and $\Phi_{j} \mu$ have compact (and thus finite) support, these operators are just multiplication operators by polynomials of finite degree in the variables $\mathrm{e}^{-i \xi_{1}}, \ldots, \mathrm{e}^{-i \xi_{d}}$, with $\xi_{j} \in(-\pi, \pi]$. So, the set $\kappa\left(H_{\mu}\right)$ is finite, and the characterisation (7.1) of the point spectrum of $H_{\mu}$ implies that $\sigma_{\mathrm{p}}\left(H_{\mu}\right)=\varnothing$ if $\operatorname{supp}(\mu) \neq\{e\}$. By taking into account Lemma 6.3.(b) and Theorem 3.6, we infer that the sets $\mathscr{D}_{t}$ are dense in $\mathcal{H}$ for each $t \geq 0$, and thus the case 2 of Section 6 applies. Finally, we mention as a corollary the following spectral result:

Corollary 7.2. Let $\mu$ be a symmetric measure on $\mathbb{Z}^{d}$ with finite support. If $\operatorname{supp}(\mu) \neq\{e\}$, then the convolution operator $H_{\mu}$ in $\mathcal{H}:=\mathrm{L}^{2}\left(\mathbb{Z}^{d}\right)$ is purely absolutely continuous.

\section{5 $H=h(P)$}

Consider in $\mathcal{H}:=\mathrm{L}^{2}\left(\mathbb{R}^{d}\right)$ the dispersive operator $H:=h(P)$, where $h \in C^{3}\left(\mathbb{R}^{d} ; \mathbb{R}\right)$ satisfies the following condition: For each multi-indices $\alpha, \beta \in \mathbb{N}^{d}$ with $\alpha>\beta,|\alpha|=|\beta|+1$, and $|\alpha| \leq 3$, we have

$$
\left|\partial^{\alpha} h\right| \leq \text { Const. }\left(1+\left|\partial^{\beta} h\right|\right) \text {. }
$$

Note that this class of operators $h(P)$ contains all the usual elliptic free Hamiltonians appearing in physics.

Take for the family $\Phi \equiv\left(\Phi_{1}, \ldots, \Phi_{d}\right)$ the position operators $Q \equiv\left(Q_{1}, \ldots, Q_{d}\right)$. Then we have for each $x \in \mathbb{R}^{d}$

$$
H(x)=\mathrm{e}^{-i x \cdot Q} H_{\mu} \mathrm{e}^{i x \cdot Q}=h(P+x),
$$


and $H^{\prime}=h^{\prime}(P)$. So Assumption 2.3 is directly verified and Assumption 2.2 follows from (7.3). Therefore all the results of the previous sections are valid. We do not give more details since many aspects of this example, including the existence of time delay, have already been extensively discussed in [37]. We only add some comments in relation with the case 3 of Section 6.

Assume that there exist $\lambda \in \mathbb{R}$ and a maximal subset $\Omega \subset \mathbb{R}^{d}$ of strictly positive Lebesgue measure such that $h(x)=\lambda$ for all $x \in \Omega$. Then any $\varphi$ in $\mathcal{H}_{\Omega}:=\{\psi \in \mathcal{H} \mid \operatorname{supp}(\mathscr{F} \psi) \subset \Omega\}$ is an eigenvector of $h(P)$ with eigenvalue $\lambda$. Furthermore, one has $\mathscr{F}^{-1} \mathcal{H}_{\Omega} \subset \mathcal{K} \equiv \operatorname{ker}\left(h^{\prime}(P)^{2}\right)$, and for simplicity we assume that the first inclusion is an equality. Then, an application of the Fourier transformation shows that $Q_{j} \mathscr{D}_{1} \subset \mathcal{G}$ for each $j$, where $\mathcal{G}$ is the orthocomplement of $\mathcal{K}$ in $\mathcal{H}$. Thus Remark 6.2 applies, and one can consider the restrictions of $H$ and $T_{f}$ to the subspace $\mathcal{G}$, as described in the case 3 of Section 6 . In favorable situations, we expect that the restriction of $T_{f}$ to $\mathcal{G}$ acts as $i \frac{\mathrm{d}}{\mathrm{d} \lambda}$ in the spectral representation of the restriction of $H$ to $\mathcal{G}$.

\subsection{Adjacency operators on admissible graphs}

Let $(X, \sim)$ be a graph $X$ with no multiple edges or loops. We write $g \sim h$ whenever the vertices $g$ and $h$ of $X$ are connected. In the Hilbert space $\mathcal{H}:=\ell^{2}(X)$ we consider the adjacency operator

$$
(H \varphi)(g):=\sum_{h \sim g} \varphi(h), \quad \varphi \in \mathcal{H}, g \in X .
$$

We denote by $\operatorname{deg}(g):=\#\{h \in X \mid h \sim g\}$ the degree of the vertex $g$. Under the assumption that $\operatorname{deg}(X):=$ $\sup _{g \in X} \operatorname{deg}(g)$ is finite, $H$ is a bounded self-adjoint operator in $\mathcal{H}$. The spectral analysis of the adjacency operator on some general graphs has been performed in [21]. Here we consider only a subclass of such graphs called admissible graphs.

A directed graph $(X, \sim,<)$ is a $\operatorname{graph}(X, \sim)$ and a relation $<$ on the graph such that, for any $g, h \in X$, $g \sim h$ is equivalent to $g<h$ or $h<g$, and one cannot have both $h<g$ and $g<h$. We also write $h>g$ for $g<h$. For a fixed $g$, we denote by $N^{-}(g) \equiv\{h \in X \mid g<h\}$ the set of fathers of $g$ and by $N^{+}(g) \equiv\{h \in X \mid$ $h<g\}$ the set of sons of $g$. The set $\{h \in X \mid g \sim h\}$ of neighbours of $g$ is denoted by $N(g) \equiv N^{-}(g) \cup N^{+}(g)$. When using drawings, one has to choose a direction (an arrow) for any edge. By convention, we set $g \leftarrow h$ if $g<h$, i.e. any arrow goes from a son to a father. When directions have been fixed, we use the simpler notation $(X,<)$ for the directed graph $(X, \sim,<)$.

Definition 7.3. A directed graph $(X,<)$ is called admissible if

(a) any closed path in $X$ has index zero (the index of a path is the difference between the number of positively oriented edges in the path and that of the negatively oriented ones),

(b) for any $g, h \in X$, one has $\#\left\{N^{-}(g) \cap N^{-}(h)\right\}=\#\left\{N^{+}(g) \cap N^{+}(h)\right\}$.

It is proved in [21, Lemma 5.3] that for admissible graphs there exists a unique (up to constant) map $\Phi: X \rightarrow \mathbb{Z}$ satisfying $\Phi(h)+1=\Phi(g)$ whenever $h<g$. With this choice of operator $\Phi$, one obtains that

$$
[H(x) \varphi](g)=\sum_{h \sim g} \mathrm{e}^{i x[\Phi(h)-\Phi(g)]} \varphi(h)
$$

for each $x \in \mathbb{R}, \varphi \in \mathcal{H}$, and $g \in X$. Therefore, the commutativity of $H$ and $H(x)$ is equivalent to the condition

$$
\sum_{h \in N(g) \cap N(\ell)}\left(\mathrm{e}^{i x[\Phi(\ell)-\Phi(h)]}-\mathrm{e}^{i x[\Phi(h)-\Phi(g)]}\right)=0
$$

for each $g, \ell \in X$. By taking into account the growth property of $\Phi$ and Hypothesis (b) of Definition 7.3, one obtains that the parts $h \in N^{-}(g) \cap N^{-}(\ell)$ and $h \in N^{+}(g) \cap N^{+}(\ell)$ of the sum are of opposite sign, and that the parts $h \in N^{-}(g) \cap N^{+}(\ell)$ and $h \in N^{+}(g) \cap N^{-}(\ell)$ are null. So Assumption 2.3 is satisfied. One also verifies by using Formula (7.4) that $H$ belongs to $C^{\infty}(\Phi)$, and that Assumption 2.2 holds. It follows that the general results presented before apply. 
that

Now, the operator $H^{\prime}$ acts as $\left(H^{\prime} \varphi\right)(g)=i\left(\sum_{h>g} \varphi(h)-\sum_{h<g} \varphi(h)\right)$, and it is proved in [21, Sec. 5]

$$
\mathcal{H}_{\mathrm{p}}(H)=\operatorname{ker}(H)=\operatorname{ker}\left(H^{\prime}\right)=\left\{\varphi \in \mathcal{H} \mid \sum_{h>g} \varphi(h)=0=\sum_{h<g} \varphi(h) \text { for each } g \in X\right\} .
$$

It is also proved that $H$ is purely absolutely continuous, except at the origin where it may have an eigenvalue with eigenspace given by (7.5). The proof of these statements is based on the method of the weakly conjugate operator [9].

However, in the present generality, it is hardly possible to obtain a simple description of the set $\kappa(H)$ or the operator $T_{f}$. We refer then to [21, Sec. 6] for explicit examples of admissible graphs with adjacency operators whose kernels are either trivial or non trivial, and develop one example for which more explicit computations can be performed. This example furnishes an illustration of the discussion in the case 3 of Section 6.

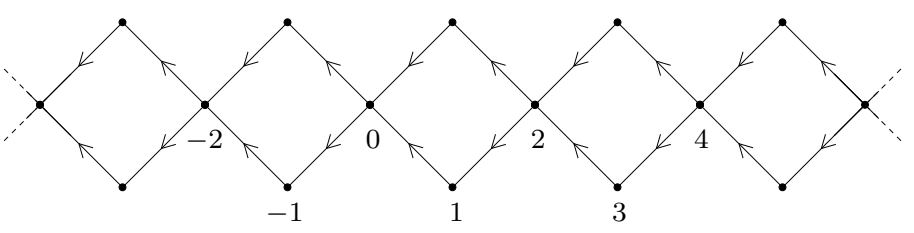

Figure 1: Example of an admissible directed graph $X$

We consider the admissible graph of Figure 1, and endow it with the function $\Phi: X \rightarrow \mathbb{Z}$ as shown on the picture. The vertices of the graph are denoted by $z_{-}$and $z_{+}$when $\Phi$ takes an odd value, and by $z$ when $\Phi$ takes an even value. More precisely, $\Phi(z)=z$ for $z$ even, and $\Phi\left(z_{-}\right)=\Phi\left(z_{+}\right)=z$ for $z$ odd. By using (7.5), it is easily observed that $\mathcal{K} \equiv \operatorname{ker}\left(\left(H^{\prime}\right)^{2}\right)$ is equal to

$$
\left\{\varphi \in \mathrm{L}^{2}(X) \mid \varphi(z)=0 \text { for } z \text { even, and } \varphi\left(z_{-}\right)=-\varphi\left(z_{+}\right) \text {for } z \text { odd }\right\} .
$$

On the other hand, the orthocomplement $\mathcal{G}$ of $\mathcal{K}$ in $\mathrm{L}^{2}(X)$ is unitarily equivalent to $\ell^{2}(\mathbb{Z})$, and the restriction $\mathrm{H}$ of $H$ to $\mathcal{G}$ is unitarily equivalent to the operator in $\ell^{2}(\mathbb{Z})$ defined by

$$
(\widetilde{\mathrm{H}} \varphi)(z):=\sqrt{2}\{\varphi(z-1)+\varphi(z+1)\}, \quad \varphi \in \ell^{2}(\mathbb{Z}) .
$$

Using the Fourier transformation, one shows that this operator is unitarily equivalent to the multiplication operator $M$ in $\mathrm{L}^{2}((-\pi, \pi])$ given by the function $(-\pi, \pi] \ni \xi \mapsto 2 \sqrt{2} \cos (\xi)$.

Now, the operator $\Phi$ in $L^{2}(X)$ is clearly reduced by the decomposition $\mathcal{K} \oplus \mathcal{G}$. As mentioned in Remark 6.2, this implies that the operator $T_{f}$ is also reduced by this decomposition. By taking Formula (7.2) into account, one obtains that the restriction $T_{f}$ of $T_{f}$ to $\mathcal{G}$ is unitarily equivalent to the operator

$$
\frac{i}{2}\left\{\frac{\mathrm{d}}{\mathrm{d} \xi}[-2 \sqrt{2} \sin (\xi)]^{-1}+[-2 \sqrt{2} \sin (\xi)]^{-1} \frac{\mathrm{d}}{\mathrm{d} \xi}\right\}
$$

on $\mathscr{F} \mathscr{D}_{1} \subset \mathrm{L}^{2}((-\pi, \pi])$. This implies, as expected, that $\mathrm{T}_{f}$ acts as $i \frac{\mathrm{d}}{\mathrm{d} \lambda}$ in the spectral representation of $\mathrm{H}$.

\subsection{Direct integral operators}

Let $\Omega$ be a measurable subset of $\mathbb{R}^{n}$ and let us consider a direct integral

$$
\mathcal{H}:=\int_{\Omega}^{\oplus} \mathrm{d} \xi \mathcal{H}_{\xi},
$$

where $\mathrm{d} \xi$ is the usual Lebesgue measure on $\mathbb{R}^{n}$ and $\mathcal{H}_{\xi}$ are Hilbert spaces. Take a decomposable self-adjoint operator $H \equiv \int_{\Omega}^{\oplus} \mathrm{d} \xi H(\xi)$ in $\mathcal{H}$. Assume that there exists a family $\Phi \equiv\left(\Phi_{1}, \ldots, \Phi_{d}\right)$ of operators in $\mathcal{H}$ such that Assumption 2.2 is satisfied. Assume also for each $x \in \mathbb{R}^{d}$ that the operator $H(x)$ defined by (2.4) is decomposable, i.e. there exists a family of self-adjoint operators $H(\xi, x)$ in $\mathcal{H}_{\xi}$ such that $H(x)=\int_{\Omega}^{\oplus} \mathrm{d} \xi H(\xi, x)$. 
Finally, assume that the operators $H(\xi)$ and $H(\xi, x)$ commute for each $x \in \mathbb{R}^{d}$ and a.e. $\xi \in \Omega$, so that $H$ and $H(x)$ commute. Then Assumption 2.3 holds, and the general theory developed in the preceding sections applies. Moreover, it is easily observed that the fibered structure of the map $x \mapsto H(x)$ implies that the operators $H_{j}^{\prime}$ are also decomposable. Therefore, there exists for each $j \in\{1, \ldots, d\}$ a family of self-adjoint operators $H_{j}^{\prime}(\xi)$ such that $H_{j}^{\prime}=\int_{\Omega}^{\oplus} \mathrm{d} \xi H_{j}^{\prime}(\xi)$. In consequence $\lambda \in \mathbb{R}$ is a regular value of $H$ if there exists $\delta>0$ and $\mathrm{C}<\infty$ such that

$$
\lim _{\varepsilon \searrow 0}\left\|\left[\left(H^{\prime}(\xi)\right)^{2}+\varepsilon\right]^{-1} E^{H(\xi)}(\lambda ; \delta)\right\|_{\mathcal{H}_{\xi}}<\mathrm{C}
$$

for a.e. $\xi \in \Omega$. We also recall that $\operatorname{ker}\left(\left(H^{\prime}\right)^{2}\right) \neq\{0\}$ if and only if there exists a measurable subset $\Omega_{0} \subset \Omega$ with positive measure such that $\operatorname{ker}\left(H^{\prime}(\xi)^{2}\right) \neq\{0\}$ for each $\xi \in \Omega_{0}$.

We now give an example of quantum waveguide-type fitting into this setting (see [35] for more details). Let $\Sigma$ be a bounded open connected set in $\mathbb{R}^{m}$, and consider in the Hilbert space $L^{2}(\Sigma \times \mathbb{R})$ the Dirichlet Laplacian $-\Delta_{\mathrm{D}}$. The partial Fourier transformation along the longitudinal axis sends the initial Hilbert space onto the direct integral $\mathcal{H}:=\int_{\mathbb{R}}^{\oplus} \mathrm{d} \xi \mathcal{H}_{0}$, with $\mathcal{H}_{0}:=\mathrm{L}^{2}(\Sigma)$, and it sends $-\Delta_{\mathrm{D}}$ onto the fibered operator $H:=\int_{\mathbb{R}}^{\oplus} \mathrm{d} \xi H(\xi)$, with $H(\xi):=\xi^{2}-\Delta_{\mathrm{D}}^{\Sigma}$. Here, $-\Delta_{\mathrm{D}}^{\Sigma}$ denotes the Dirichlet Laplacian in $\Sigma$. By Choosing for $\Phi$ the position operator $Q$ along the longitudinal axis one obtains that $H(x)=\int_{\mathbb{R}}^{\oplus} \mathrm{d} \xi H(\xi, x)$ with $H(\xi, x)=$ $(\xi+x)^{2}-\Delta_{\mathrm{D}}^{\Sigma}$. Clearly, $H(\xi)$ and $H(\xi, x)$ commute, and so do $H$ and $H(x)$. Furthermore, the operator $H$ is of class $C^{\infty}(\Phi)$, and $H^{\prime}$ is the fibered operator given by $H^{\prime}(\xi)=2 \xi$. It follows that both Assumptions 2.2 and 2.3 hold, and thus the general theory applies. Now a simple calculation using (7.6) shows that $\kappa(H)=\sigma\left(-\Delta{ }_{\mathrm{D}}^{\Sigma}\right)$. Furthermore, in the tensorial representation $\mathrm{L}^{2}(\Sigma) \otimes \mathrm{L}^{2}(\mathbb{R})$ of $\mathrm{L}^{2}(\Sigma \times \mathbb{R})$, one obtains that $T_{f}=T=\frac{1}{4} \otimes$ $\left(Q P^{-1}+P^{-1} Q\right)$ on the dense set

$$
\mathscr{D}_{1}=\left\{\varphi \in \mathrm{L}^{2}(\Sigma) \otimes \mathcal{D}(\langle Q\rangle) \mid \varphi=\eta\left(-\Delta_{\mathrm{D}}\right) \varphi \text { for some } \eta \in C_{\mathrm{c}}^{\infty}(\mathbb{R} \backslash \kappa(H))\right\},
$$

and $T_{f}$ is equal to $i \frac{\mathrm{d}}{\mathrm{d} \lambda}$ in the spectral representation of $-\Delta_{\mathrm{D}}$. In [35] it is even shown that the quantum time delay exists and is given by Formula (6.4) for appropriate scattering pairs $\left\{-\Delta_{\mathrm{D}},-\Delta_{\mathrm{D}}+V\right\}$.

\section{Acknowledgements}

S. Richard is supported by the Swiss National Science Foundation. R. Tiedra de Aldecoa is partially supported by the Núcleo Científico ICM P07-027-F "Mathematical Theory of Quantum and Classical Magnetic Systems" and by the Chilean Science Foundation Fondecyt under the Grant 1090008.

\section{References}

[1] W. O. Amrein, A. Boutet de Monvel and V. Georgescu. $C_{0}$-groups, commutator methods and spectral theory of $N$-body Hamiltonians, volume 135 of Progress in Math. Birkhäuser, Basel, 1996.

[2] W. O. Amrein and M. B. Cibils. Global and Eisenbud-Wigner time delay in scattering theory. Helv. Phys. Acta 60: 481-500, 1987.

[3] W. O. Amrein, M. B. Cibils and K. B. Sinha. Configuration space properties of the $S$-matrix and time delay in potential scattering. Ann. Inst. Henri Poincaré 47: 367-382, 1987.

[4] W. O. Amrein and Ph. Jacquet. Time delay for one-dimensional quantum systems with steplike potentials. Phys. Rev. A 75(2): 022106, 2007.

[5] I. Antoniou, I. Prigogine, V. Sadovnichii and S. A. Shkarin. Time operator for diffusion. Chaos Solitons Fractals 11(4): 465-477, 2000.

[6] A. Arai. Generalized Weyl relation and decay of quantum dynamics. Rev. Math. Phys. 17(9): 1071-1109, 2005. 
[7] M. Sh. Birman and M. Z. Solomjak. Spectral theory of selfadjoint operators in Hilbert space. Mathematics and its Applications (Soviet Series). D. Reidel Publishing Co., Dordrecht, 1987. Translated from the 1980 Russian original by S. Khrushchëv and V. Peller.

[8] A. Boutet de Monvel and V. Georgescu. The method of differential inequalities. In Recent developments in quantum mechanics pp. 279-298. Math. Phys. Stud. 12, Kluwer Acad. Publ., Dordrecht, 1991.

[9] A. Boutet de Monvel, G. Kazantseva and M. Măntoiu. Some anisotropic Schrödinger operators without singular spectrum. Helv. Phys. Acta 69(1): 13-25, 1996.

[10] E. B. Davies. Spectral theory and differential operators, volume 42 of Cambridge Studies in Advanced Mathematics Cambridge University Press, Cambridge, 1995.

[11] G. B. Folland. A course in abstract harmonic analysis. Studies in Advanced Mathematics. CRC Press, Boca Raton, 1995.

[12] E. A. Galapon. Pauli's theorem and quantum canonical pairs: the consistency of a bounded, self-adjoint time operator canonically conjugate to a Hamiltonian with non-empty point spectrum. R. Soc. Lond. Proc. Ser. A Math. Phys. Eng. Sci. 458: 451-472, 2002.

[13] V. Georgescu and C. Gérard. On the virial theorem in quantum mechanics. Commun. Math. Phys. 208: 275-281, 1999.

[14] F. Gómez. Self-adjoint time operators and invariant subspaces. Rep. Math. Phys. 61(1): 123-148, 2008.

[15] C. Gérard and R. Tiedra de Aldecoa. Generalized definition of time delay in scattering theory. J. Math. Phys. page 122101, 2007.

[16] T. Gotō, K. Yamaguchi and N. Sudō. On the time operator in quantum mechanics. Three typical examples. Progr. Theoret. Phys. 66(5): 1525-1538, 1981.

[17] F. Hiroshima, S. Kuribayashi and Y. Matsuzawa. Strong time operators associated with generalized Hamiltonians. Lett. Math. Phys. 87(1-2): 115-123, 2009.

[18] P. T. Jørgensen and P. S. Muhly. Selfadjoint extensions satisfying the Weyl operator commutation relations. J. Analyse Math. 37: 46-99, 1980.

[19] M. Miyamoto. A generalized Weyl relation approach to the time operator and its connection to the survival probability. J. Math. Phys. 42(3): 1038-1052, 2001.

[20] A. Mohapatra, K. B. Sinha and W. O. Amrein. Configuration space properties of the scattering operator and time delay for potentials decaying like $|x|^{-\alpha}, \alpha>1$. Ann. Inst. H. Poincaré Phys. Théor. 57(1): 89-113, 1992.

[21] M. Măntoiu, S. Richard and R. Tiedra de Aldecoa. Spectral analysis for adjacency operators on graphs. Ann. Henri Poincaré 8(7): 1401-1423, 2007.

[22] M. Măntoiu and R. Tiedra de Aldecoa. Spectral analysis for convolution operators on locally compact groups. J. Funct. Anal. 253(2): 675-691, 2007.

[23] J. G. Muga and C. R. Leavens. Arrival time in quantum mechanics. Phys. Rep. 338(4): 353-438, 2000.

[24] J. G. Muga, R. Sala Mayato and Í. L. Egusquiza, editors. Time in quantum mechanics. Vol. 1, volume 734 of Lecture Notes in Physics. Springer, Berlin, second edition, 2008.

[25] M. Razavy. Time of arrival operator. Canad. J. Phys. 49: 3075-3081, 1971.

[26] M. Reed and B. Simon. Methods of modern mathematical physics I, Functional analysis. Academic Press Inc. [Harcourt Brace Jovanovich Publishers], New York, second edition, 1980. 
[27] M. Reed and B. Simon. Methods of modern mathematical physics II, Fourier analysis, Self-adjointness. Academic Press Inc. [Harcourt Brace Jovanovich Publishers], New York, 1975.

[28] J. Riss. Eléments de calcul différentiel et théorie des distributions sur les groupes abéliens localement compacts. Acta Math. 89: 45-105, 1953.

[29] D. Robert and X. P. Wang. Existence of time-delay operators for Stark Hamiltonians. Comm. Partial Differential Equations 14(1): 63-98, 1989.

[30] D. Robert and X. P. Wang. Time-delay and spectral density for Stark Hamiltonians. II. Asymptotics of trace formulae. Chinese Ann. Math. Ser. B 12(3): 358-383, 1991.

[31] J. Sahbani. The conjugate operator method for locally regular Hamiltonians. J. Operator Theory 38(2): 297-322, 1997.

[32] J. Sahbani. Spectral theory of certain unbounded Jacobi matrices. J. Math. Anal. Appl. 342: 663-681, 2008.

[33] K. Schmüdgen. On the Heisenberg commutation relation. I. J. Funct. Anal. 50(1): 8-49, 1983.

[34] B. Thaller. The Dirac Equation. Springer-Verlag, Berlin, 1992.

[35] R. Tiedra de Aldecoa. Time delay and short-range scattering in quantum waveguides. Ann. Henri Poincar é 7(1): 105-124, 2006.

[36] R. Tiedra de Aldecoa. Anisotropic Lavine's formula and symmetrised time delay in scattering theory. Math. Phys. Anal. Geom. 11(2): 155-173, 2008.

[37] R. Tiedra de Aldecoa. Time delay for dispersive systems in quantum scattering theory. Rev. Math. Phys. 21(5): 675-708, 2009.

[38] J. Weidmann. Linear operators in Hilbert spaces. Springer-Verlag, New York, 1980.

[39] Z.-Y. Wang and C.-D. Xiong. How to introduce time operator. Ann. Physics 322(10): 2304-2314, 2007. 